\title{
MASSES AND ORBITAL CONSTRAINTS FOR THE OGLE-2006-BLG-109Lb,c JUPITER/SATURN ANALOG
} PLANETARY SYSTEM

\author{
D. P. Bennett ${ }^{1}$, S. H. Rhit ${ }^{1}$, S. Nikolaev ${ }^{2}$, B. S. Gaudi ${ }^{3}$, A. Udalski ${ }^{4}$, A. Gould ${ }^{3}$, G. W. Christie ${ }^{5}$, D. Maoz ${ }^{6}$, S. Dong ${ }^{3}$, \\ J. McCormick ${ }^{7}$, M. K. Szymański ${ }^{4}$, P. J. Tristram ${ }^{8}$, B. Macintosh ${ }^{2}$, K. H. CoOK ${ }^{2}$, M. KUbiak ${ }^{4}$, G. PietrzyŃski ${ }^{4,9}$, \\ I. SOSZYŃSKI ${ }^{4}$, O. SZEWCZYK ${ }^{9}$, K. ULACZYK ${ }^{4}$, Ł. WYRZYKOWSKI ${ }^{4,10}$ \\ (The OGLE Collaboration)

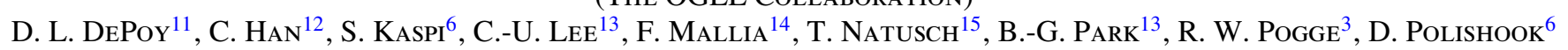 \\ (The $\mu$ FUN COLlaboration) \\ F. Abe ${ }^{16}$, I. A. Bond ${ }^{17}$, C. S. BotZler ${ }^{18}$, A. Fukui ${ }^{16}$, J. B. Hearnshaw ${ }^{19}$, Y. Itow ${ }^{16}$, K. KAmiYA ${ }^{16}$, A. V. Korpela ${ }^{20}$, \\ P. M. Kilmartin ${ }^{8}$, W. Lin $^{17}$, J. Ling ${ }^{17}$, K. Masuda ${ }^{16}$, Y. Matsubara ${ }^{16}$, M. Motomura $^{16}$, Y. Muraki ${ }^{21}$, S. Nakamura ${ }^{16}$, \\ T. Okumura ${ }^{16}$, K. Ohnishi ${ }^{22}$, Y. C. Perrott ${ }^{18}$, N. J. Rattenbury ${ }^{18}$, T. Sako ${ }^{16}$, To. Saito ${ }^{23}$, S. Sato ${ }^{24}$, L. Skuljan ${ }^{18}$, \\ D. J. Sullivan ${ }^{20}$, T. Sumi ${ }^{16}$, W. L. Sweatman ${ }^{17}$, P. C. M. Yock ${ }^{18}$ \\ (The MOA COllaboration) \\ M. Albrow ${ }^{19}$, A. Allan ${ }^{25}$, J.-P. Beaulieu ${ }^{26}$, D. M. Bramich ${ }^{27}$, M. J. BurgdorF ${ }^{28,29}$, C. Coutures ${ }^{26}$, M. Dominik ${ }^{30}$,

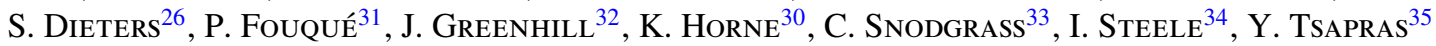

(From the Planet and RoboNet Collaborations)

B. Chaboyer ${ }^{36}$, A. CROCKER ${ }^{37}$, AND S. Frank ${ }^{3}$

${ }^{1}$ Department of Physics, University of Notre Dame, Notre Dame, IN 46556, USA; bennett@nd.edu

${ }^{2}$ IGPP, Lawrence Livermore National Laboratory, 7000 East Avenue, Livermore, CA 94550, USA

${ }^{3}$ Department of Astronomy, Ohio State University, 140 West 18th Avenue, Columbus, OH 43210, USA

${ }^{4}$ Warsaw University Observatory, Al. Ujazdowskie 4, 00-478 Warszawa, Poland

${ }^{5}$ Auckland Observatory, P.O. Box 24-180, Auckland, New Zealand

${ }^{6}$ Faculty of Exact Sciences, School of Physics and Astronomy, Tel-Aviv University, Tel Aviv 69978, Israel

${ }^{7}$ Farm Cove Observatory, 2/24 Rapallo Place, Pakuranga, Auckland 1706, New Zealand

${ }^{8}$ Mount John Observatory, P.O. Box 56, Lake Tekapo 8770, New Zealand

${ }^{9}$ Departamento de Fisica, Universidad de Concepción, Casilla 160-C, Concepción, Chile

${ }^{10}$ Institute of Astronomy, University of Cambridge, Madingley Road, Cambridge CB3 0HA, UK

${ }^{11}$ Department of Physics \& Astronomy, Texas A\&M University, College Station, TX 77843, USA

12 Department of Physics, Chungbuk National University, 410 Seongbong-Rho, Hungduk-Gu, Chongju 371-763, Korea

${ }^{13}$ Korea Astronomy and Space Science Institute, 61-1 Hwaam-Dong, Yuseong-Gu, Daejeon 305-348, Korea

${ }^{14}$ Campo Catino Astronomical Observatory, P.O. Box Guarcino, Frosinone 03016, Italy

${ }^{15}$ School of Computing and Mathematical Sciences, AUT University, Auckland, New Zealand

${ }^{16}$ Solar-Terrestrial Environment Laboratory, Nagoya University, Nagoya 464-8601, Japan

${ }^{17}$ Institute for Information and Mathematical Sciences, Massey University, Auckland 1330, New Zealand

${ }_{18}^{18}$ Department of Physics, University of Auckland, Private Bag 92-019, Auckland 1001, New Zealand

${ }^{19}$ Department of Physics and Astronomy, University of Canterbury, Christchurch 8020, New Zealand

${ }^{20}$ School of Chemical and Physical Sciences, Victoria University, Wellington, New Zealand

${ }^{21}$ Department of Physics, Konan University, Nishiokamoto 8-9-1, Kobe 658-8501, Japan

${ }^{22}$ Nagano National College of Technology, Nagano 381-8550, Japan

${ }^{23}$ Tokyo Metropolitan College of Aeronautics, Tokyo 116-8523, Japan

${ }^{24}$ Faculty of Science, Department of Physics and Astrophysics, Nagoya University, Nagoya 464-8602, Japan

${ }^{25}$ School of Physics, University of Exeter, Stocker Road, Exeter, EX4 4QL, UK

${ }^{26}$ Institut d'Astrophysique de Paris, CNRS, 98bis Boulevard Arago, 75014 Paris, France

${ }^{27}$ European Southern Observatory, Karl-Schwarzschild-Straße 2, 85748 Garching bei München, Germany

${ }^{28}$ SOFIA Science Center, NASA Ames Research Center, Mail Stop N211-3, Moffett Field, CA 94035, USA

${ }^{29}$ Deutsches SOFIA Institut, Universitaet Stuttgart, Pfaffenwaldring 31, 70569 Stuttgart, Germany

${ }^{30}$ School of Physics \& Astronomy, SUPA, University of St. Andrews, North Haugh, St. Andrews, KY16 9SS, UK

${ }^{31}$ LATT, CNRS, Université de Toulouse, 14 avenue Edouard Belin, 31400 Toulouse, France

${ }^{32}$ School of Mathematics and Physics, University of Tasmania, Private Bag 37, Hobart, TAS 7001, Australia

${ }^{33}$ European Southern Observatory, Casilla 19001, Vitacura 19, Santiago, Chile

${ }^{34}$ Astrophysics Research Institute, Liverpool John Moores University, Egerton Wharf, Birkenhead CH41 1LD, UK

${ }^{35}$ Las Cumbres Observatory, 6740B Cortona Dr, suite 102, Goleta, CA 93117, USA

${ }^{36}$ Department of Physics and Astronomy, Dartmouth College, 6127 Wilder Laboratory, Hanover, NH 03755, USA

${ }^{37}$ Department of Astronomy, University of Massachusetts, Amherst, MA 01002, USA

Received 2009 November 17; accepted 2010 March 2; published 2010 March 26

\begin{abstract}
We present a new analysis of the Jupiter+Saturn analog system, OGLE-2006-BLG-109Lb,c, which was the first double planet system discovered with the gravitational microlensing method. This is the only multi-planet system discovered by any method with measured masses for the star and both planets. In addition to the signatures of two planets, this event also exhibits a microlensing parallax signature and finite source effects that provide a direct measure of the masses of the star and planets, and the expected brightness of the host star is confirmed by Keck AO imaging, yielding masses of $M_{*}=0.51_{-0.04}^{+0.05} M_{\odot}, M_{b}=231 \pm 19 M_{\oplus}$, and $M_{c}=86 \pm 7 M_{\oplus}$. The Saturn-analog planet in this system had a planetary light-curve deviation that lasted for 11 days, and as a result, the effects of the orbital motion are visible in the microlensing light curve. We find that four of the six orbital
\end{abstract}


parameters are tightly constrained and that a fifth parameter, the orbital acceleration, is weakly constrained. No orbital information is available for the Jupiter-analog planet, but its presence helps to constrain the orbital motion of the Saturn-analog planet. Assuming co-planar orbits, we find an orbital eccentricity of $\epsilon=0.15_{-0.10}^{+0.17}$ and an orbital inclination of $i=64^{\circ}{ }_{-7^{\circ}}$. The $95 \%$ confidence level lower limit on the inclination of $i>49^{\circ}$ implies that this planetary system can be detected and studied via radial velocity measurements using a telescope of $\gtrsim 30 \mathrm{~m}$ aperture.

Key words: gravitational lensing: micro - planetary systems

\section{INTRODUCTION}

The discovery of the Jupiter/Saturn analog planetary system, OGLE-2006-BLG-109Lb,c, by gravitational microlensing (Gaudi et al. 2008) suggests that solar systems like our own may be common, at least among systems that contain gas giant planets. This was generally assumed to be the case prior to the detection of the first extrasolar planets orbiting main sequence stars (Mayor \& Queloz 1995; Marcy \& Butler 1996; Butler \& Marcy 1996), but these first discoveries challenged this conventional wisdom, with the discovery of hot-Jupiters and gas giants in highly eccentric orbits. However, the discovery of true solar system analogs with the radial velocity method is difficult, requiring radial velocity precision $<3 \mathrm{~m} \mathrm{~s}^{-1}$ spanning a decade or more (Wright et al. 2008), so it may be that the apparent paucity of systems like our own is a selection effect.

The gravitational microlensing method has very different selection effects from the radial velocity method. It is most sensitive to planets at separations similar to the Einstein radius, which in most cases is just beyond the "snow line" at $\sim 2.7 M / M_{\odot}$ (Ida \& Lin 2004; Lecar et al. 2006; Kennedy et al. 2006; Kennedy \& Kenyon 2008) where gas giant planets are expected to form, according to the core accretion theory. Microlensing does find that super-Earth and Neptune-mass planets (Beaulieu et al. 2006; Gould et al. 2006; Bennett et al. 2008) are more common at these separations than Jupiters. Of the handful of microlensing events that reveal gas giant planets (Bond et al. 2004; Udalski et al. 2005), only OGLE-2006-BLG-109 and MOA-2007-BLG400 (Dong et al. 2009b) are highly sensitive to multiple planets of Jupiter mass or less. The fact that OGLE-2006-BLG-109 did reveal a Jupiter-Saturn-like system suggests that systems like our own are common among stars hosting gas giants near or beyond the snow line.

The OGLE-2006-BLG-109L system is the first multi-planet system found by microlensing but several other aspects of this event are also unique. The light curve reveals the microlensing parallax effect, which yields a geometric mass measurement of the planetary host star and both of the planets. In addition, the host star is 5 times brighter than the source in the $H$ band, and it is detected in Keck AO images. The measured $H$-band brightness confirms the microlensing parallax mass measurement.

In addition, the light-curve shape is sensitive to the relative positions of the planet at the pico-arcsecond level, and the lightcurve signal of the Saturn-mass planet is visible for 11 days. As a result, the orbital velocity of this planet in the plane of the sky must be included to model the light curve. Moreover, these effects also provide a weak constraint on the orbital acceleration.

In this paper, we present some details of the analysis that was summarized by Gaudi et al. (2008), and a new analysis of OGLE-2006-BLG-109 that includes the orbital motion of the OGLE-2006-BLG-109L system. With the assumption of co-planar planetary orbits and orbital stability constraints, we derive limits on the full set of orbital parameters for this Saturnmass planet.
This paper is organized as follows. In Section 2, we discuss the image data and the photometric reductions. The modeling of the light curve is described in Section 3, and a new method for determining the angular radii of the source star and the Einstein ring is presented in Section 4. The follow-up observations that identify the planetary host star and their analysis are discussed in Section 6, and Section 7 discusses possible alternative models for the light curve. Section 8 discusses the orbital motion modeling and the conversion from measured fit parameters to inferred orbital parameters, and the Bayesian analysis used to find the constraints on the physical orbital parameters is discussed in Section 9. Limits on additional planets in the system are discussed in Section 10, and we conclude in Section 11.

\section{LIGHT-CURVE DATA AND PHOTOMETRY}

Microlensing event OGLE-2006-BLG-109 (right ascension $\alpha=17^{\mathrm{h}} 52^{\mathrm{m}} 34.51$, declination $\delta=-30^{\circ} 05^{\prime} 16^{\prime \prime} \cdot 0$, J2000) was announced by the Optical Gravitational Lensing Experiment (OGLE) Collaboration Early Warning System (EWS; Udalski et al. 1994) on 2006 March 26 (heliocentric Julian day 3821), triggering follow-up observations by the Microlensing Observations in Astrophysics (MOA) Collaboration using the $0.61 \mathrm{~m}$ telescope at Mount John Observatory in New Zealand. Two days later, the OGLE Early-Early Warning System (EEWS; Udalski 2003) detected a deviation from the standard single lens light curve. This led OGLE to take three additional images of the field that includes this event on the same night. These additional images confirmed the deviation, and so OGLE issued an anomaly alert. The OGLE alert message noted that "short-lived, low amplitude" anomalies can be caused by planetary companions, and implied that there was a good chance that this was planetary signal. This suggestion would prove to be correct, although in this case, the planets orbiting the lens star would generate additional large amplitude anomalies over the subsequent 10 days.

This anomaly alert prompted follow-up observations from the Microlensing Follow-up Network ( $\mu$ FUN), Probing Lensing Anomalies Network (PLANET) and Robonet, although relatively few PLANET and Robonet telescopes were available for bulge observations so early in the season. Data were obtained from 11 different telescopes spanning the globe. The two telescopes providing data from Chile were the OGLE Warsaw $1.3 \mathrm{~m}$ ( $I$ band) and the Cerro Tololo Inter-American Observatory (CTIO) Small and Moderate Aperture Research Telescope System (SMARTS) $1.3 \mathrm{~m}(V, I$, and $H$ bands) telescopes. From the southwestern U.S., data were provided through the $\mu$ FUN Collaboration from the MDM $2.4 \mathrm{~m}$ ( $I$ band) and the Mount Lemmon $1.0 \mathrm{~m}$ ( $I$ band) telescopes in Arizona, as well as the Aero8 $0.3 \mathrm{~m}$ (unfiltered) telescope in New Mexico, operated by the Campo Catino Astronomical Observatory. Data from New Zealand came from the MOA $0.61 \mathrm{~m}$ telescope ( $I$ band) at the Mount John Observatory on the South Island, and the Auckland $0.35 \mathrm{~m}$ and Farm Cove $0.25 \mathrm{~m}$ telescopes, which are both $\mu$ FUN telescopes located in the vicinity of Auckland. Slightly 
further west is the PLANET Canopus $1.0 \mathrm{~m}$ telescope ( $I$ band) in Tasmania, Australia.

The most sparsely covered longitudes are those west of Australia, and east of Chile, where only Northern Hemisphere telescopes were available to observe this event. The two telescopes filling this gap were the $1.0 \mathrm{~m}$ Wise telescope (unfiltered) in Israel, associated with $\mu \mathrm{FUN}$, and the Robonet/Liverpool $2.0 \mathrm{~m}$ telescope ( $R$ band) at La Palma in the Canary Islands.

Although it is very difficult to get complete light-curve coverage with these telescopes so early in the Galactic bulge observing season, we were able to obtain good coverage of four of the five caustic crossing or cusp approach features in the light curve. Much of the first feature was missed before the light-curve anomaly was detected. Three of the remaining four features were visible from New Zealand, where the availability of telescopes on both the North and South Islands allowed good coverage of these three features even though each observatory missed one anomaly due to bad weather.

An additional anomaly that would prove to be caused by a second planet was observed from the Wise Observatory in Israel and by OGLE and $\mu$ FUN-CTIO in Chile.

The photometry of the OGLE data and most of the $\mu \mathrm{FUN}$ data were re-processed after the event was over using the OGLE pipeline (Udalski 2003). The exceptions were the CTIO $H$-band data, which were reduced with DOPHOT (Schechter et al. 1993), and the Wise data, which were reduced using SoDOPHOT (Bennett et al. 1993). The MOA photometry was done with the MOA difference imaging pipeline (Bond 2000), and the PLANET-Canopus and RoboNET-Liverpool Telescope data were reduced with Pysis (Albrow et al. 2009).

The color dependence of atmospheric extinction can significantly affect the unfiltered (or "clear" filter) photometry taken by some of the smaller telescopes due to the variation of the airmass toward the target throughout the night (Dong et al. 2009a). This color dependence of the extinction causes apparent variation in the relative photometry of stars of different colors as the airmass changes. Since DIA photometry is effectively normalized to some average of the brighter stars in the field, this can introduce systematic photometry errors. This can be corrected by ensuring that the photometry of the target star is normalized to stars with the same color as the source star. The "clear" filter data sets that cover important parts of the light curve are the Auckland, Farm Cove, and Wise data sets. For these data sets, we have determined the $V-I$ colors of all the bright stars in the images by matching the stars found in SoDOPHOT reductions of the clear filter data sets to $V$ - and $I$-band images from CTIO. The light curves of these stars were then generated using the OGLE pipeline for the Auckland and Farm Cove data and SoDOPHOT for the Wise data. A weighted mean of these bright star light curves with the same average color as the target star was then used to normalize each OGLE-2006-BLG-109 light curve.

This normalization procedure was complicated by the fact that the OGLE-2006-BLG-109 source star is blended with a relatively bright clump giant star, as discussed in Section 6, which is slightly redder than the source star. The separation between the source and this blended star is only 0.'35, so these stars are never resolved in these clear filter images. Thus, the photometry is being done on a blended target that changes color as the lensing magnification changes. This complicates the normalization scheme for the unfiltered photometry as described above, because the color of the blended image of the two stars is changing with time. However, we expect this effect to be small because the difference between the source and blend colors is only $\sim \Delta(V-I)=0.2$. Nevertheless, we estimate the color of the combined source-plus-blend image based on an early light-curve model and find that the correction is indeed small. Of course, the exact values of the correction do depend somewhat on the arbitrary choice of which model is used to make the correction but, in fact, the correction is so small that any reasonable model will give the same result to much better than one-tenth of the estimated errors.

We have followed the standard microlensing analysis procedure of rescaling the error bars in order to obtain $\chi^{2} /$ dof for each data set. Each data point had an assumed systematic error of $0.3 \%$ added in quadrature to the error reported by the photometry code, and then the error bars were rescaled by the following factors: 1.5 for OGLE, 1.42 for MOA, 1.31 for CTIO $I$ band, 1.14 for CTIO $H$ band, 1.55 for MDM, 0.52 for Mount Lemmon, 1.93 for Canopus, 2.3 for the Liverpool telescope, 1.8 for Auckland, 1.04 for Farm Cove, 1.5 for Wise, and 1.0 for Campo Catino.

The light-curve modeling described in Section 3 requires limb darkening parameters because finite source effects are important. The analysis of the extinction and color of the source in Section 4 implies that the source has an approximate spectral type of G0, with $T_{\text {eff }} \approx 6000 \mathrm{~K}$, and the radius estimate implies that $\log g \approx 4.5$. From Claret (2000), this implies linear limb darkening parameters of $u_{V}=0.6630$, $u_{R}=0.5887, u_{I}=0.5090$, and $u_{H}=0.3292$. For the unfiltered Farm Cove and Auckland data, $u_{F C}=0.5413$, and $u_{\text {Auck }}=0.5490$ are the preferred values. These were estimated from color transformations derived using the method of Gould et al. (2010). The method indicates that the unfiltered or "clear" Farm Cove and Auckland passbands can be represented as $C_{\mathrm{FC}}=0.79 I+0.21 \mathrm{~V}$ and $C_{\text {Auck }}=0.74 I+0.26 \mathrm{~V}$, and we have estimated the linear limb darkening coefficients with these same linear transformations using the $I$ - and $V$-band linear coefficients from Claret (2000). We used $u_{R}$ for the Wise data set. Most of the modeling was actually done with an earlier estimate of the source temperature, $T_{\text {eff }} \approx 6250 \mathrm{~K}$ and an older compilation of the limb darkening parameters (Diaz-Cordoves et al. 1995; Claret et al. 1995). The values used for most of the modeling runs were $u_{V}=0.633, u_{R}=0.535, u_{I}=0.456, u_{H}=0.275$, $u_{F C}=0.502$, and $u_{\text {Auck }}=0.493$. The newer limb darkening parameters yield a $\chi^{2}$ improvement of $\Delta \chi^{2}=0.61$ and have no significant effect on any of the model or derived parameters.

\section{LIGHT-CURVE MODELING}

OGLE-2006-BLG-109 is the first double-planet microlensing event and the most complicated microlensing event yet to be successfully modeled. The strongly magnified portion of the light curve is shown in Figure 1, with the peak region of the light curve shown in Figure 2 and close-ups of the five causticcrossing and cusp approach features shown in Figure 3. The light-curve models shown in these figures have 15 nonlinear parameters plus 24 linear parameters that describe the source flux and background flux in each of the 12 instrumental passbands. These linear parameters can be solved for exactly once the other 15 parameters are specified, but determination of these 15 nonlinear parameters is highly non-trivial.

Two of these 15 parameters describe microlensing parallax (Refsdal 1966; Gould 1992; Alcock et al. 1995) and three more describe the orbital motion of one of the planets. The parallax parameters generate small perturbations in the light 


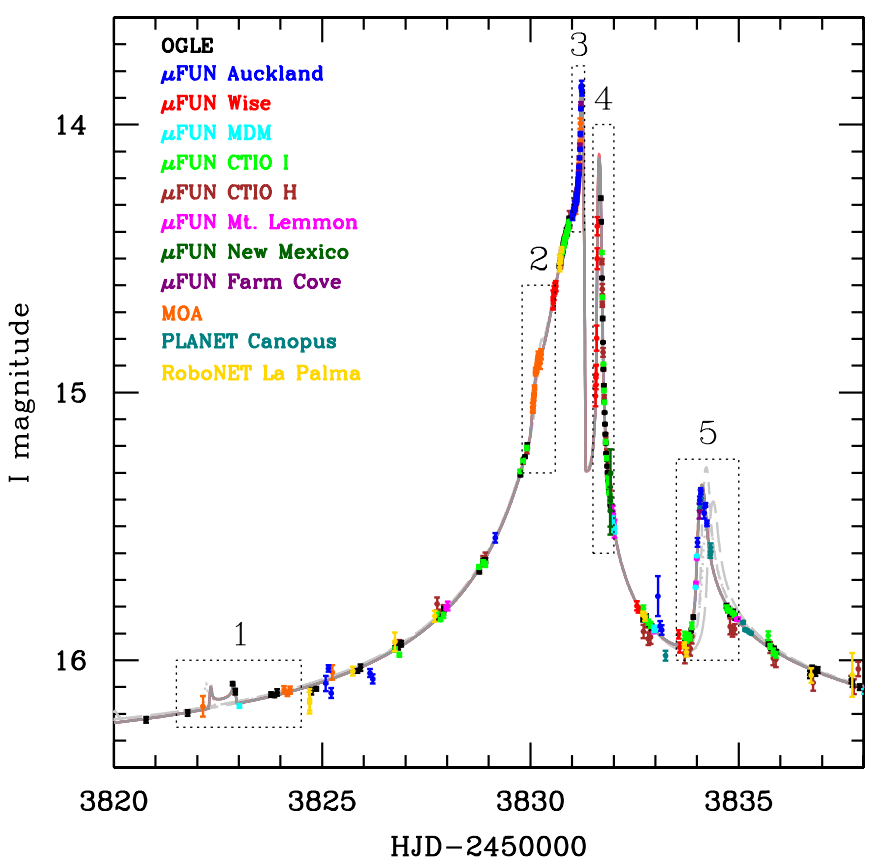

Figure 1. Photometric measurements and model light curves are shown for 18 days near the peak of the OGLE-2006-BLG-109 light curve, with telescope and passband for each data point indicated by its color, as indicated. The best-fit model is plotted as a solid gray curve, and the light gray curves plotted with dots, short-dashes, and long-dashes indicate the same model, but with parallax, orbital motion, and both parallax and orbital model removed, respectively. (These alternative models are more easily seen in Figures 1 and 2.) The five caustic crossing and cusp approach or crossing features are indicted by the dotted boxes labeled $1-5$.

curve over long timescales, as well as very small perturbations near peak magnification (Hardy \& Walker 1995; Holz \& Wald 1996), and the orbital motion parameters provide relatively small perturbations to the timing and shape of the various light-curve features. So, it is sensible to initially ignore these parameters, and to try and find an approximate solution with the remaining ten parameters. These ten parameters include the three parameters of single lens events, the Einstein radius crossing time, $t_{E}$, the separation of closest approach between the source and the lens system center of mass, $u_{0}$, and the time of this closest approach, $t_{0}$. An additional parameter, the source radius crossing time, $t_{*}$, is needed for a small fraction of single lens events and most planetary system lens events to describe the finite source effects. There are six additional parameters that describe the static configuration. The mass fractions of the two planets are $\epsilon_{1}$ and $\epsilon_{2}$, and $\epsilon_{3}=1-\epsilon_{1}-\epsilon_{2}$ is the mass fraction of the planetary host star. The angle between the source trajectory and the line connecting mass- 1 to the center of mass of masses 2 and 3 is $\theta_{1 \mathrm{~cm}}$, and the distance between mass- 1 and the mass $2+3$ center of mass is $d_{1 \mathrm{~cm}}$. The remaining parameters are the distance between masses 2 and $3, d_{23}$ and the angle between the line connecting these two masses with respect to the line between mass 1 and the mass $2+3$ center of mass, $\phi_{23}$. Both $d_{1 \mathrm{~cm}}$ and $d_{23}$ are measured in Einstein radius units.

There are two basic aspects involved in modeling planetary microlensing events: the method used to calculate the microlensing light curve and the strategy employed to locate the appropriate model in the multi-dimensional parameter space. The lightcurve calculations were done using variations of the method first introduced by Bennett \& Rhie (1996), who developed the first general binary-lens, finite source light-curve calculation code and used it to demonstrate the sensitivity of the microlensing

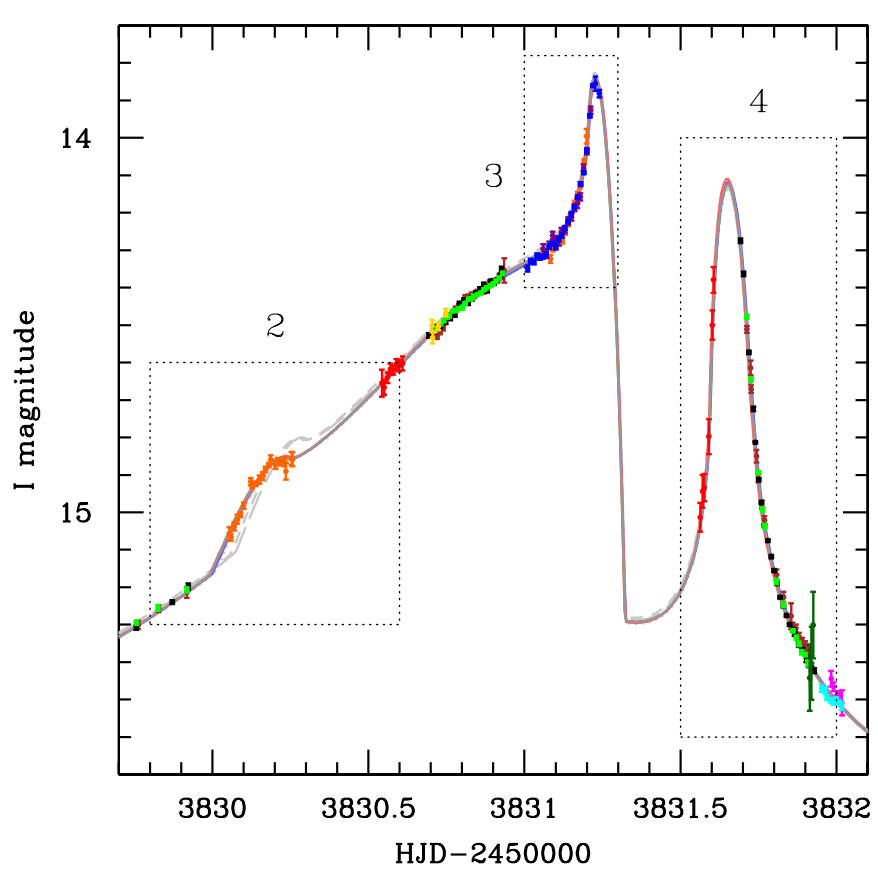

Figure 2. Close-up of the OGLE-2006-BLG-109 light-curve peak showing caustic crossing and cusp approach features 2-4. OGLE-2006-BLG-109 lightcurve peak. As in Figure 1, the light gray curves plotted with dots, short-dashes, and long-dashes indicate the best-fit model without parallax, orbital motion, and both parallax and orbital motion, respectively, while the solid curve(s) indicate the best-fit model.

method to low-mass planets. Their method uses the point-source approximation except when the source is in the vicinity of a caustic curve, where an inverse ray-shooting integration scheme is used. The point-source calculations for triple lens models are done using the method of Rhie (2002). At a preliminary stage of the calculation, one of the sub-groups doing the modeling also used the binary lens superposition approximation (Rhie \& Bennett 1996; Bozza 1999; Rattenbury et al. 2002; Han 2005; Kubas et al. 2008).

The search for solutions for triple-lens systems is substantially more difficult than for binary-lens models due to the additional three parameters needed to describe the second planet. The method of Bennett (2009) is particularly well suited to such events. It uses a grid only for the initial conditions, and then allows all parameters to vary while minimizing $\chi^{2}$ from these initial points. This allows the solution to be found in a fully automated manner without a huge increase in the computation time due to the larger dimension of parameter space. As we shall see, this method is also relatively efficient for modeling events that include lens orbital motion. For this event, however, the modeling effort is aided by the fact that the major features in the light curve are covered reasonably well and by the fact that the features due to the different planets do not overlap on the light curve. So, the global parameter search part of the Bennett (2009) method was not used.

The light-curve modeling began while the event was still in progress, and modeling done after the detection of features 1 , 2 , and 3 shown in Figures 1-3 was able to predict the future behavior of the event, and in particular feature 5 (Gaudi et al. 2008). However, this model did not predict feature 4. Because the light-curve deviations due to multiple planets often resemble the superposition of the planetary deviations due to each planet alone (Rhie \& Bennett 1996; Bozza 1999; Rattenbury et al. 2002; Han 2005), this was considered a strong hint that the signal 

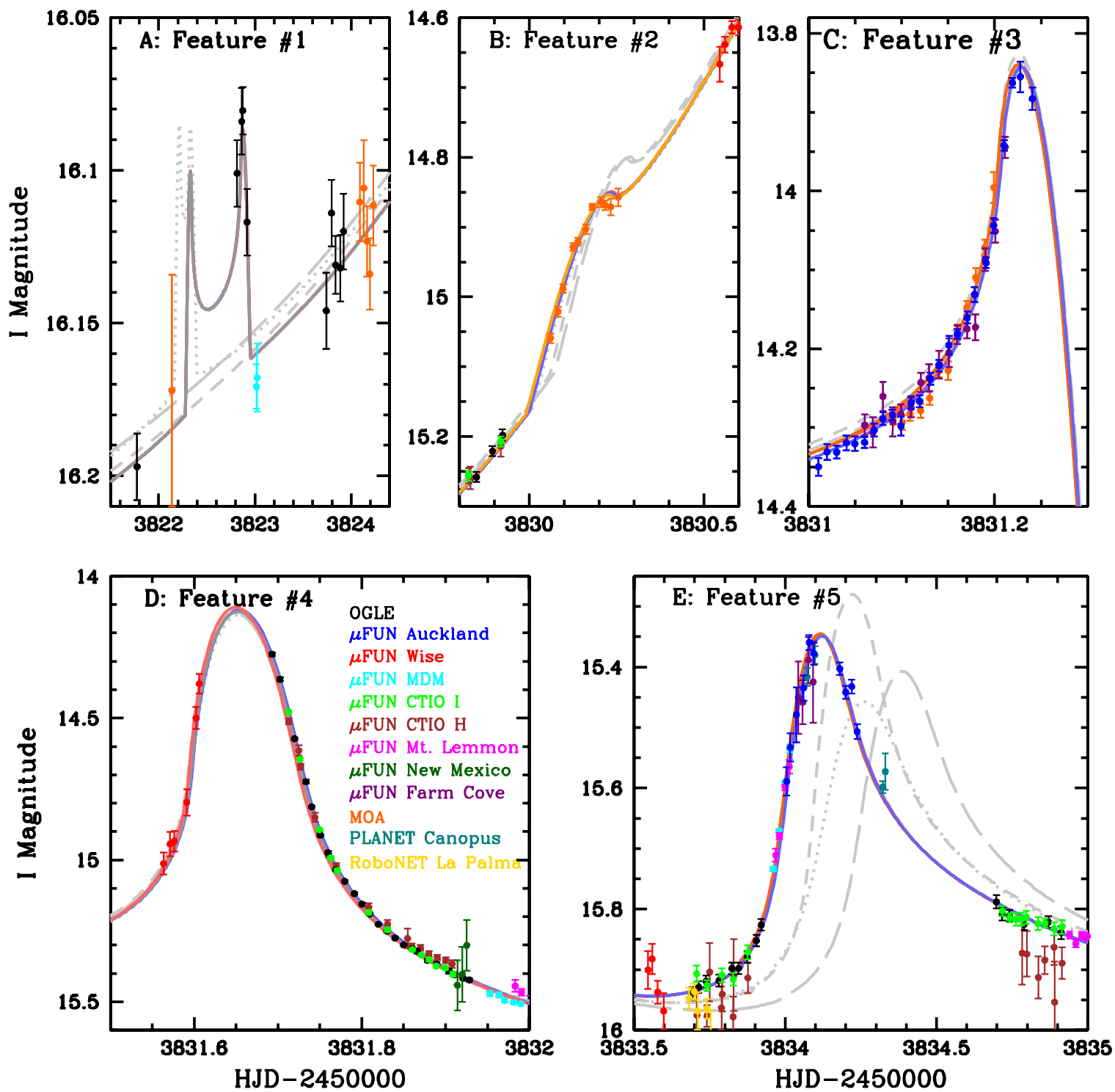

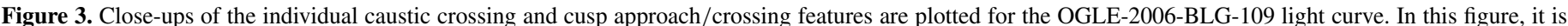

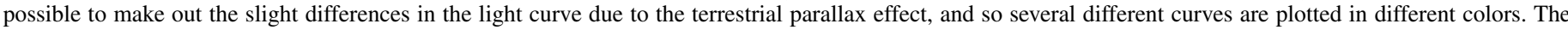

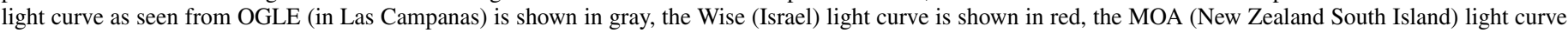

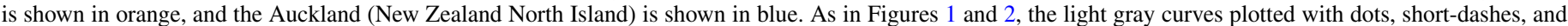
long-dashes indicate the best-fit model without parallax, orbital motion, and both parallax and orbital motion, respectively.

of a second planet was present. Nevertheless, two subgroups proceeded with systematic attempts to model this event with a single planet model using methods similar to that of Cassan (2008), but developed independently. Attempts were made to find binary lens models that could explain four of the five features, and it was found that this was only possible when the fourth feature was excluded, just as the preliminary analysis during the event had indicated.

The binary superposition approximation (Han 2005) with Markov Chain Monte Carlo (MCMC) minimization was then used to search for approximate static, two-planet solutions. This search succeeded in identifying classes of models that could explain the basic features of the light curve. A second group then used this class of models as an approximate input to a full triple lens modeling code (Rhie 2002; Bennett et al. 1999) and the light-curve calculation and Metropolis et al. (1953) $\chi^{2}$ minimization recipe of Bennett (2009). The initial fitting using static, triple-lens models could almost explain the OGLE-2006-
BLG-109 light curve, but it proved impossible to fit features 1 and 5 with the same static lens model.

However, the typical orbital motion of a planet in a microlensing event is about $10^{-3} R_{E}$ per day, and for an event like OGLE2006-BLG-109, for which the main signal comes from a planet near the Einstein ring, the radial motion of the caustic has an amplitude similar to, but slightly larger than the motion of the planet. (The angular motion of the caustic is much smaller than this.) As a result, over the 11 days between features 1 and 5, we can expect that the radial position of the caustic curve will change by $\sim 0.01 R_{E}$, which is more than enough to have a significant effect on the light curve. So, it was obvious that the orbital motion of the planet close the Einstein ring would have a significant effect on the light curve, and the failure of the static, triple-lens models indicated that orbital motion must be included.

The inclusion of orbital motion adds a significant complication to the modeling of microlensing events. For most 
high-magnification microlensing events, it is possible to significantly reduce the light-curve computation time by making use of the fact that the lens configuration stays approximately the same throughout the event. With this static approximation, it is possible to make and store a single map of the two-dimensional magnification of the lens as a function of source position, from which one can quickly and efficiently draw many trial one-dimensional light curves, thus reusing the information for many different observations. This is the basis of the inverse ray-shooting (Wambsganss 1997; Rattenbury et al. 2002) and magnification map (Dong et al. 2006) methods, which densely ray-shoot broad swaths of the image plane to determine the magnification of a large number of source positions. In particular, the calculations of high-magnification events light curves can be made substantially more efficient in this way by only ray-shooting the images near the Einstein ring. Unfortunately, these shortcuts cannot be used for modeling an event with orbital motion because the lens configuration is different for every observation.

The Bennett \& Rhie (1996) method also uses a version of the ray-shooting method for its finite source calculations, but rather than sampling the entire image plane, simply samples those images that are created for a given source position. The current version of this method (Bennett 2009) allows the option to store the rays shot in the vicinity of the Einstein ring, so that they do not have to be re-calculated for different observations, but this optimization cannot be used for this event. This method also employs some numerical integration improvements that speed up these high-magnification event calculations by a large factor $(\sim 100)$. So, despite the fact that the orbital motion prevents the ray-storage optimization, the method of Bennett (2009) has been proved efficient enough to model this event.

The importance of orbital motion can be seen in Figure 4, which shows a time series of caustic positions at intervals of 2.9 days. The red curve is the caustic position at the time of feature 1 . It reveals the two caustic crossing seen in the lightcurve model. The second caustic entrance (feature 2) occurs about a day before the time of the green caustic curve, and the highest magnification peak of the light curve occurs on the caustic exit (feature 3 ) at the time of the black caustic curve. The final cusp approach (feature 5) occurs at the time of the blue caustic curve.

It is apparent that the radial motion of the caustic in Figure 4 is much larger than the rotation of the caustic. This is, in fact, a general feature of orbital motion in high-magnification microlensing events. This orbital motion is most easily detected when a planet is close to the Einstein ring, so that the caustic is extended and the planetary signal has a long duration. But in this situation, the radial motion of the central caustic has a velocity similar to the velocity of the planet. However, the angular motion of the caustic is smaller than the planet's angular velocity by the distance of the caustic from the origin (center of mass) in Einstein radius units. For this lens system, the caustic extends to a distance of $\sim 0.15$ Einstein radii from the center of mass, but the source does not encounter the caustic at this furthest point. With the actual source trajectory, the caustic is encountered at a distance of $\sim 0.05$ Einstein radii, and so the angular motion of the caustic is suppressed by a factor of 20 compared to the radial motion. This situation is typical, so we should generally expect that the effect of the angular motion of the planet in the plane of the sky will usually be compressed by a factor of $\sim 10$ compared to the apparent radial motion. Thus, it will usually not be useful to approximate orbital motion as rotation in the plane of the sky for high magnification events, although this is

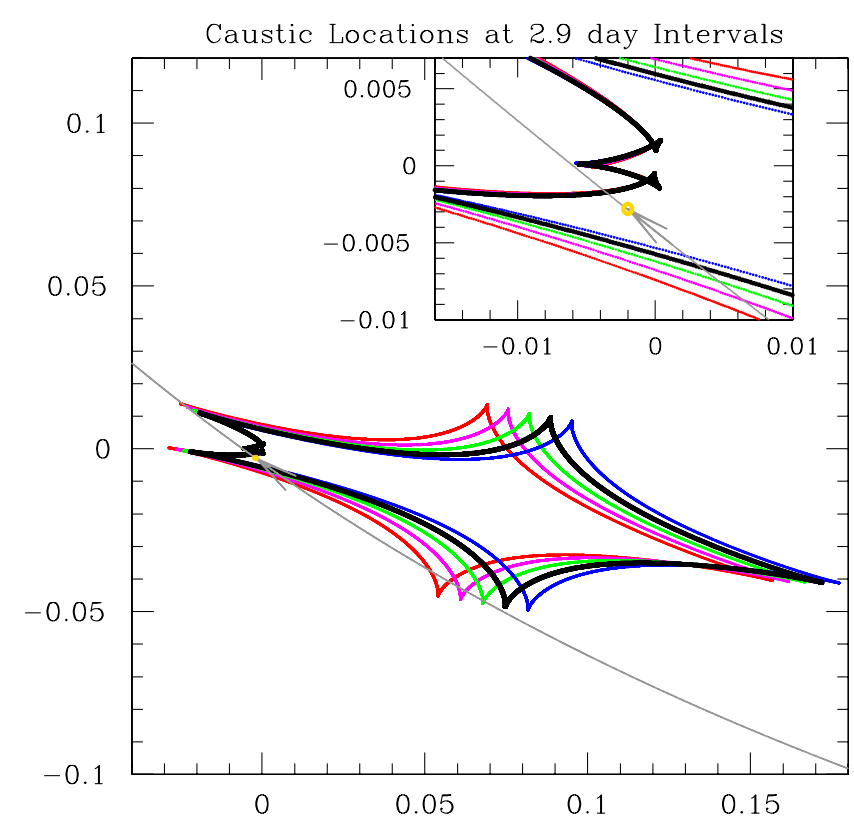

Figure 4. Configuration of the central (resonant) caustic curves for OGLE2006-BLG-109 is shown at five different times at intervals of 2.9 days ranging from $t=3822.5$, which is the time of the first caustic crossing, to $t=3834.1$, which is the time of the final cusp approach. The red, magenta, green, black, and blue curves represent $t=3822.5,3825.4,3828.3,3831.2$, and 3834.1, respectively. The gray curve is the source trajectory, which is curved due to microlensing parallax (i.e., the motion of the Earth). The inset shows a close-up of the central region of the central caustic, which includes the triangular-shaped portion that is due to the Jupiter-mass planet, OGLE-2006-BLG-109Lb. The gold circle indicates the angular size of the source star.

much more efficient to investigate computationally (Rattenbury et al. 2002).

The basic procedure used to find the solution for this event has been to proceed from a simple single-planet plus star model without orbital motion, and then to add additional effects oneby-one. This is done in the following order:

1. A static model with a star and a single planet, approximately matching all features, except feature 4.

2. Add a second planet to account for feature 4. The best static two-planet model cannot simultaneously explain the details of features 1 and 5 .

3. Include orbital motion of the Saturn-mass planet, which is responsible for features 1-3 and 5 .

4. Add microlensing parallax to include the effect of the orbital motion of the Earth.

The orbital motion of the Saturn-like planet, like that of any other mass, can be described by seven parameters. These include six parameters, such as a three-dimensional position and velocity, that can describe the initial conditions, plus the mass of the system, which is required to determine the future orbital motion. For an object in a Keplerian orbit, the conventional parameters include parameters like eccentricity and time of periapsis, which are not likely to be well constrained by a microlensing light curve, when the planetary signal only lasts 11 days. So, we have selected a set of parameters that can be separated according to how well they are constrained by the microlensing light curve. The two-dimensional position of the planet in the plane of the sky is described by two of the static lens-system parameters. The lowest order effect of orbital motion is simply the two-dimensional velocity of the planet in the plane of the sky described by $\dot{d}_{23 x}$ and $\dot{d}_{23 y}$ (where the $x$ direction is defined by the line from mass 1 to the mass 2-3 
center of mass). For the models we consider here, the Jupiteranalog planet is mass 1 , the Saturn analog planet is mass 2, and the host star is mass 3, so it is masses 2 and 3 that are in orbital motion.

The two-dimensional position and velocity of the Saturnanalog planet (with respect to the host star) gives us four parameters (out of seven total) that are tightly constrained. The simplest possibility would simply be to use a constant velocity for the Saturn-mass planet, and ignore any higherorder terms such as the acceleration of the planet. Although this approximation does not correspond to a consistent orbital solution, it is reasonable for events for which orbital motion is only weakly detected, where the higher-order terms are not constrained (Dong et al. 2009a). However, there are a couple of problems with adopting this approach. One danger of such a strategy is that this unphysical motion could have a significant effect elsewhere in the light curve. For example, the planetary caustic could move close enough to the source trajectory to be detected. While it is unlikely that this would happen for the bestfit model, it is more likely to happen for somewhat disfavored models that may be explored when we determine the parameter uncertainties with long MCMC runs.

Another disadvantage of this unphysical parameterization scheme is that it is possible that other parameters, beyond the two-dimensional velocities, will be constrained by the lightcurve data, and in fact, we find that this is indeed the case for OGLE-2006-BLG-109. However, in order to minimize the number of unconstrained parameters, we do not permit the full freedom that orbital motion allows. Instead, we restrict the orbital motion to circular orbits and add a single additional parameter, the orbital period, $T_{\text {orb }}$.

The next light-curve feature to be included is microlensing parallax. This can be described (Gould 2000; Bennett 2008) by the projected Einstein radius, $\tilde{\mathbf{r}}_{\mathbf{E}}$, which has a magnitude, $\tilde{r}_{E}$, equal to the Einstein radius projected (from the source) to the position of the observer and a direction parallel to the lens-source relative proper motion. This can be measured in a variety of ways. The most common way to measure $\tilde{r}_{E}$ is through the effect of the orbital motion of the Earth (Gould 1992; Alcock et al. 1995), but it has also been measured via the spatial separation of different telescopes on the Earth (Hardy \& Walker 1995; Holz \& Wald 1996; Gould et al. 2009) and via observations from satellites in heliocentric orbit (Dong et al. 2007). For OGLE-2006-BLG-109, we find that the orbital parallax effect is dominant, as the event is quite long with $t_{E}>120$ days, but we also see a significant terrestrial parallax effect, due to the different locations of the observatories on the Earth. However, this terrestrial parallax was not included in the initial modeling reported in Gaudi et al. (2008).

While it is most convenient to describe microlensing parallax in terms of the projected Einstein radius vector, $\tilde{\mathbf{r}}_{\mathbf{E}}$, this is not a convenient parameter to use for microlensing light-curve fits. It is often the case that the microlensing parallax signal is weak, but this implies that $\tilde{r}_{E} \rightarrow \infty$. So, it is conventional to use the microlensing parallax vector, $\boldsymbol{\pi}_{E} \| \tilde{\mathbf{r}}_{\mathbf{E}}$, which has a magnitude, $\pi_{E}=1 \mathrm{AU} / \tilde{r}_{E}$. As advocated by Gould (2004), we work in a geocentric frame, which is at rest with respect to the Earth at a fixed time (HJD $=2,453,831$ days in this case). We use polar coordinates, $\pi_{E}$ and $\phi_{E}$ to describe $\pi_{E}$, so that the north and east components of $\pi_{E}$ are $\pi_{E, N}=\pi_{E} \cos \phi_{E}$ and $\pi_{E, E}=\pi_{E} \sin \phi_{E}$.

The addition of microlensing parallax yields 15 nonlinear parameters needed to model this event. In addition, there are two linear parameters for each telescope and passband to describe the source flux and blended (or background) flux for each of these data sets. These linear parameters are solved for exactly for each set of nonlinear parameters that is considered.

With these 15 parameters, we can now find a fit that explains all the features of the OGLE-2006-BLG-109 light-curve, but this is not enough to ensure that we have found the best solution. In particular, the planetary orbital motion and the microlensing parallax can change the relative motion of the source and the caustic curves in a similar way. Thus, it is possible that our minimization routine and our MCMC runs will not fully explore the degeneracy in these parameters. Therefore, we have done a series of $\chi^{2}$ minimization runs with fixed values for $\pi_{E}$ ranging from $\pi_{E}=0.216$ to $\pi_{E}=0.515$, which map out the trade-off between parallax and orbital velocity and ensure that we have found the true $\chi^{2}$ minimum.

For single lens and binary lens events, there is usually an approximate four-fold degeneracy in the microlensing parallax solutions (Smith et al. 2003; Gould 2004), although this degeneracy can be broken for very long events or events with a significant terrestrial parallax signal. One of these degeneracies is completely broken for triple lens events as it is related to the reflection symmetry about the lens axis that exists for binary lens events. The third mass breaks this reflection symmetry, so that only one approximate symmetry remains. This remaining symmetry involves reflecting all of the lens system parameters with respect to the parallax parameters, and then shifting the parallax parameters by an amount that depends on the time of year when the event occurs. (In terms of the model parameters, this symmetry implies $u_{0} \rightarrow-u_{0}, \theta_{1 \mathrm{~cm}} \rightarrow-\theta_{1 \mathrm{~cm}}$, and $\phi_{23} \rightarrow-\phi 23$.) With the inclusion of terrestrial parallax, we find that this degenerate parallax solution is disfavored by $\Delta \chi^{2}=37.9$, so that it is not a viable solution.

For high magnification events, there is often also a degeneracy associated with the $d \rightarrow 1 / d$ transformation that occurs when the source only probes the "central caustic" created by a planet, whose shape is approximately invariant under this transformation (Griest \& Safizadeh 1998). This degeneracy does not apply to the Saturn-mass planet because it is close to the Einstein ring. The source is observed while crossing some of the "planetary parts" of the caustic curve, which is not invariant under this transformation. However, this degeneracy does apply to the Jupiter-mass planet, which is not so close to the Einstein ring. In Gaudi et al. (2008), we argued that the $d_{J}<1$ solution was disfavored by $\Delta \chi^{2}=11.4$ over the $d_{J}>1$ solution. However, the inclusion of terrestrial parallax improves the $\chi^{2}$ of the best $d_{J}<1$ solution by 12.0 and the $\chi^{2}$ of the best $d_{J}>1$ solution by 22.0. So, the $d_{J}>1$ solutions do now seem nearly as good as the $d_{J}<1$ solutions. However, as we will see in Section 8, the parameters of these $d_{J}>1$ solutions do not generally correspond to planets with stable, co-planar orbits. As a result, the $d_{J}<1$ solutions remain strongly favored.

\section{SOURCE STAR RADIUS AND ANGULAR EINSTEIN RADIUS}

The angular radius of the source star, $\theta_{*}$, is an important parameter, because we can combine it with the Einstein radius crossing time and the source radius crossing time to yield the angular Einstein radius,

$$
\theta_{E}=\frac{\theta_{*} t_{E}}{t_{*}},
$$




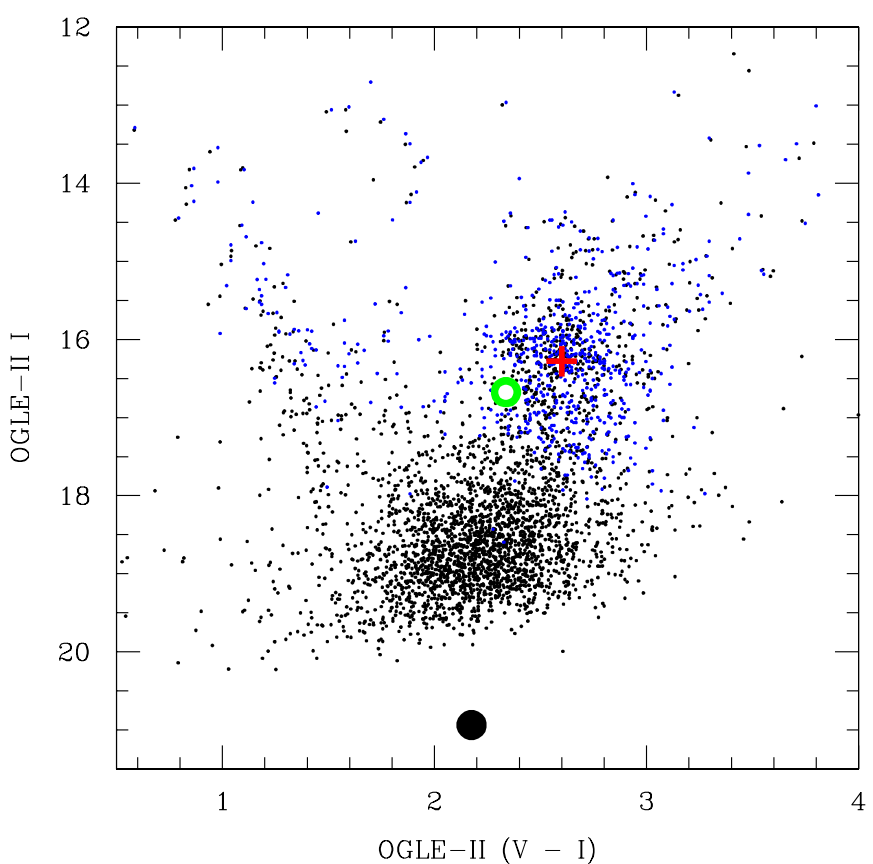

Figure 5. CMD of the stars within $60^{\prime \prime}$ of the OGLE-2006-BLG-109 source star in $V$ - and $I$-band magnitudes calibrated to the OGLE-II system. The small black dots indicate the OGLE-II photometry, and the blue dots indicate the CTIO photometry that has been calibrated to the OGLE-II system and matched with the CTIO $H$-band photometry (which does not go as deep as $V$ and $I$ ). The red cross indicates the inferred centroid of the "red clump," the green open circle indicates the location of the bright star 0.'35 from the source star, and the large black dot indicates the location of the source star.

which is needed to determine the masses of the lens star and its planets.

The angular radius or the source is routinely estimated from $V$ - and I-band data (Yoo et al. 2004), but the availability of $\mathrm{H}$-band photometry that is precise enough to yield an accurate $H$-band magnitude for the source allows a much more precise method that uses three-color VIH photometry. This method is more precise because it allows us to take into account slight spatial variations in the extinction and differing extinction laws toward different lines of sight, and because it allows us to use the more precise optical-IR surface brightness relations (Kervella et al. 2004). The $V-H$ relation has a precision of $1.1 \%$, to be compared to the nonlinear $V-I$ relations, which has a precision of about 5\% (Kervella \& Fouqué 2008).

The first step toward estimating the intrinsic source star color and magnitude is to identify the centroid of the red clump star distribution in color-magnitude diagrams (CMD) made of stars in the vicinity of the target star and then to compare these colors and magnitudes to the known properties of these red clump giant stars. We take the absolute red clump star magnitudes for the local stellar population, with Hipparcos parallaxes, from Alves et al. (2002): $M_{K}=-1.60 \pm 0.03, M_{I}=-0.26 \pm 0.03$, and $M_{V}=0.73 \pm 0.03$. However, while red clump stars are approximate standard candles, their properties are known to vary with age, metallicity, and chemical composition. Salaris \& Girardi (2002) have calculated these corrections to the red clump magnitudes for the known age and metallicity of giant stars in the Galactic bulge. They present two sets of Galactic bulge corrections, one assuming a standard solar chemical abundance distribution and another for $\alpha$-enhanced abundances, which is expected to be more appropriate for the Galactic bulge. We use the $\alpha$-enhanced corrections, but we increase the error bars

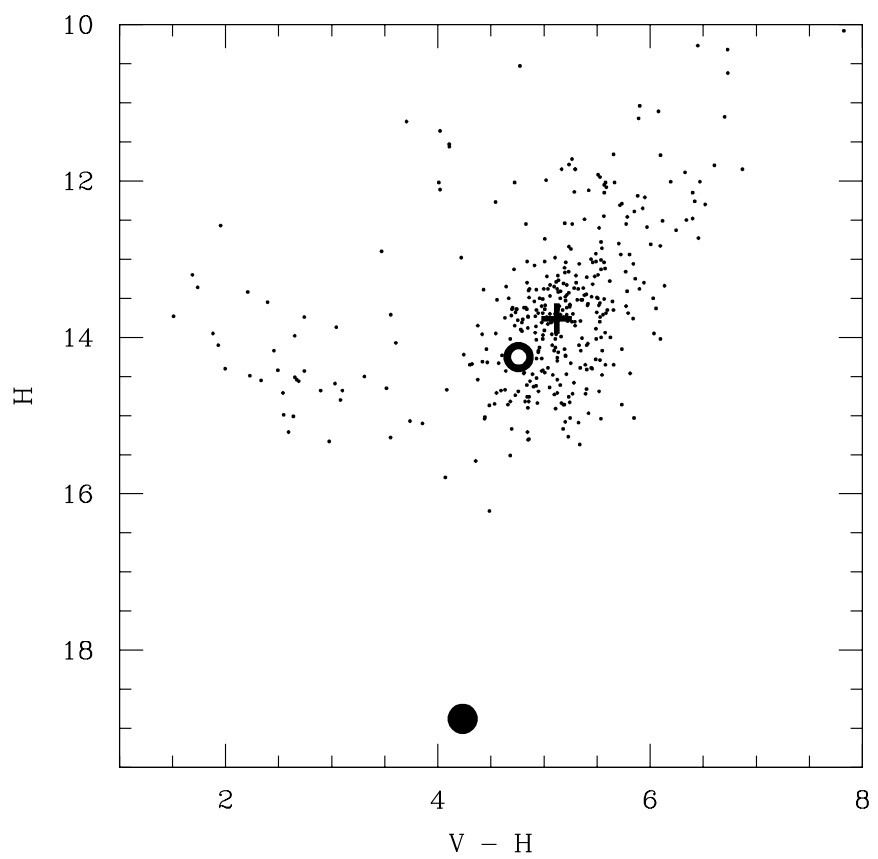

Figure 6. $V-H \mathrm{CMD}$ of the stars within $60^{\prime \prime}$ of the OGLE-2006-BLG-109 source star, based upon $V$ - and $H$-band magnitudes from the Andicam instrument on the CTIO $1.3 \mathrm{~m}$ telescope. The $V$ band has been calibrated to the OGLE-II system, and the $H$ band has been calibrated to 2MASS. As in Figure 5, the cross indicates the inferred centroid of the "red clump," the open circle indicates the location of the bright star 0.'35 from the source star, and the large black dot indicates the location of the source star.

in proportion to the difference between the $\alpha$-enhanced and solar metallicity corrections to account for uncertainties in this correction and the possibility of spatial dependence in the chemical composition of bulge red clump stars. This gives the following estimates of the absolute magnitude of the red clump:

$$
\begin{aligned}
& M_{\mathrm{Krc}}=-1.49 \pm 0.03, \\
& M_{\mathrm{Hrc}}=-1.41 \pm 0.04, \\
& M_{\mathrm{Irc}}=-0.25 \pm 0.05, \\
& M_{\mathrm{Vrc}}=0.79 \pm 0.08,
\end{aligned}
$$

where we have used the Bessell \& Brett (1988) giant star color-color relations to derive the $H$-band magnitude.

These magnitudes must now be compared to the observed red clump magnitudes in the vicinity of the OGLE-2006-BLG-109S source star. Figures 5-7 show CMDs of all the stars within 1' of OGLE-2006-BLG-109S. Figure 5 uses $V$ and $I$ magnitudes from both OGLE-II and CTIO. The CTIO magnitudes are only shown for stars that are identified in all three bands observed with the CTIO-ANDICAM instrument: $V, I$, and $H$. Since the $H$-band images are relatively shallow, the three-band CTIO photometry (shown as blue dots in Figure 5) does not extend to stars much fainter than the red clump. The small black dots indicate the OGLE-II photometry, which is not constrained to have stellar counterpart identified in the $H$-band data. The source star and the centroid of the red clump star distribution are indicated by the large black and red dots, respectively, and the location of the bright star that is $0 ! 35$ from the source is indicated by the large green dot. 


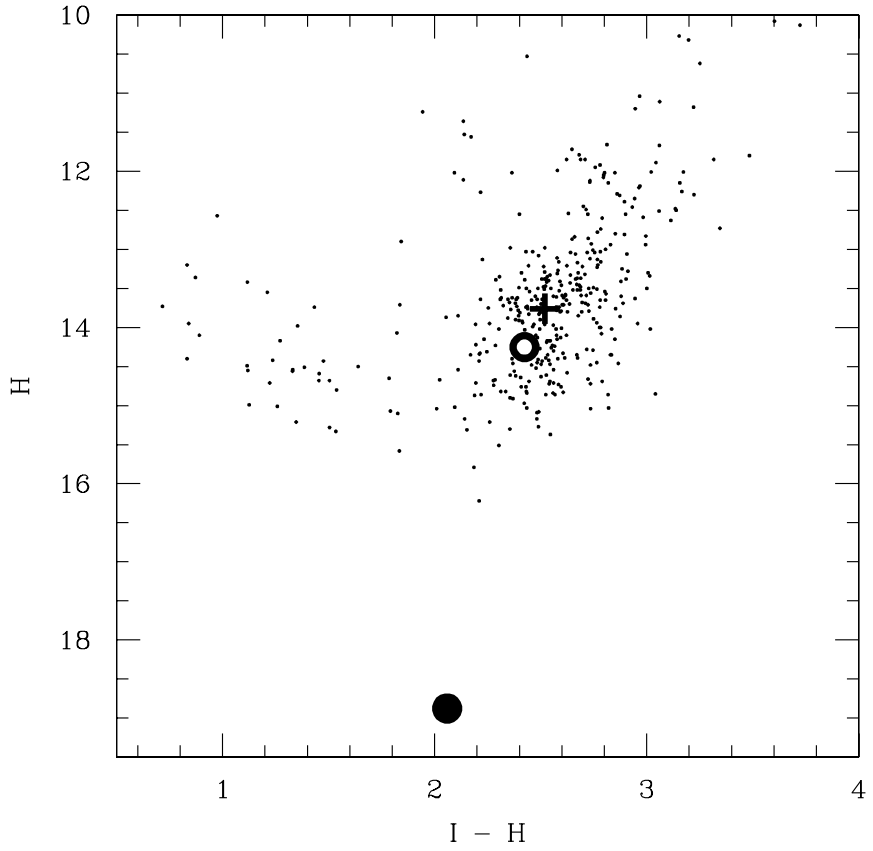

Figure 7. $I-H \mathrm{CMD}$ of the stars within $60^{\prime \prime}$ of the OGLE-2006-BLG-109 source star, based on the data described in Figures 5 and 6.

The $V$ and $I$ magnitudes from CTIO have been calibrated to the same OGLE-II system used for the OGLE data (Udalski et al. 2002), and the CTIO $H$-band data have been calibrated to 2MASS (Carpenter 2001) ${ }^{38}$ using a set of common stars that have been found to have no close neighbors in the higher angular resolution CTIO frames.

We locate the centroids of the red clump distribution in these CMDs by first creating a smoothed stellar density distribution by convolving each CMD with a two-dimensional Gaussian with $\sigma=0.1 \mathrm{mag}$. We then find the maximum of the red clump distribution in this smoothed CMD. This was done for each of the CMDs plotted in Figures 5-7, including both the CTIO and OGLE-II $V-I$ CMDs shown in Figure 5.

We also make a correction to the clump position measured in the field of OGLE-2006-BLG-109S due to crowding. The need for such a correction is apparent from artificial star tests of photometry in crowded stellar fields (Alcock et al. 2001). The photometry codes used to make the magnitude measurements for the CMDs are less complete in finding faint stars that are located under the point-spread functions (PSFs) of brighter stars. So, these faint stars are more likely to be detected when they are not in the vicinity of brighter stars. Thus, the unresolved star background is, on average, brighter under the PSFs of bright stars. Since these photometry codes do not account for this, they tend to slightly overestimate the brightnesses of these brighter stars. The appropriate correction can be estimated from the OGLE-II analysis of microlensed red clump stars by Sumi et al. (2006). The source brightnesses for these are determined by the microlens model fit, and so they are independent of this photometric bias. Considering only the 29 stars from Sumi et al. (2006) with source flux fractions $f_{s}>0.5$, we find $\left\langle f_{s}\right\rangle=0.95 \pm 0.03$. (Stars with $f_{s}>0.5$ are generally blends of two giant stars and do not appear to be part of the clump.) This is a weighted average, but we have added an error of 0.1 in quadrature to the fit uncertainty reported by Sumi et al.

\footnotetext{
${ }^{38}$ Improved calibrations are available at

http://www.ipac.caltech.edu/2mass/releases/allsky/doc/sec6_4b.html.
}

(2006) to prevent the shot noise from the few events with very small $f_{s}$ fit uncertainties from increasing the uncertainties. This correction might have some color dependence as the red clump stars are likely to have a color that is somewhat different than the stars responsible for this blending effect. However, we only have an estimate of this effect in the $I$ band, so we add 0.05 mag correction to the VIH magnitude values determined from the CMDs. The errors in this procedure are dominated by the uncertainty in locating the clump centroid in the CMDs. We tried a variety of different smoothing radii to find the centroids for all four CMDs shown in Figures 5-7. (Figure 5 has CMDs from both OGLE-II and CTIO.) The clump centroids for all CMDs with all reasonable smoothing radii are consistent with these adopted red clump magnitudes and uncertainties,

$$
\begin{gathered}
H_{\mathrm{rc}}=13.76 \pm 0.10, \\
I_{\mathrm{rc}}=16.28 \pm 0.10, \\
V_{\mathrm{rc}}=18.88 \pm 0.10,
\end{gathered}
$$

for the red clump stars within $1^{\prime}$ of OGLE-2006-BLG-109S.

These measured red clump magnitudes allow a comparison of the predicted absolute red clump magnitudes given in Equations (3)-(5) with the measured magnitudes from Equations (6)-(8). We now fit for the extinction assuming the Cardelli et al. (1989) extinction law, and a Galactic center distance of $R_{0}=8.0 \pm 0.2 \mathrm{kpc}$. This model has two parameters, and there are three constraints, the VIH magnitudes of the red clump centroid. The best fit yields $A_{V}=3.47 \pm 0.07$ and $R_{V I}=A_{V} /\left(A_{V}-A_{I}\right)=2.41_{-0.19}^{+0.24}$. These parameters also imply that $A_{I}=2.03 \pm 0.08, A_{H}=0.60 \pm 0.04$ and $R_{V}=2.96_{-0.46}^{+0.70}$. With two model parameters and three measurements, we have $\chi^{2}=0.151$ for a single degree of freedom. We note that the Cardelli et al. (1989) law uses an infrared extinction law that does not quite agree with direct measurements toward the Galactic bulge (Nishiyama et al. 2006). However, since we are only considering one infrared passband (the $H$ band), we are not forcing infrared extinction to follow the Cardelli law between different infrared passbands. So, this deficiency of the Cardelli law will have little influence on our results.

If we assume that the source star has the same extinction as the average of the red clump stars within $1^{\prime}$ of the source, then we can use the Kervella et al. (2004) $(V-H, H)$ color-radius relations to derive a source radius of $0.474_{-0.018}^{+0.020} \mu$ as. Note that these Kervella relations use infrared magnitudes that are effectively on the Bessell \& Brett (1988) system, so we must convert from the Two Micron All Sky Survey (2MASS) system to Bessell \& Brett using the formulae in Carpenter (2001).

However, the extinction toward the source is not identical to the average extinction toward the clump giants within $1^{\prime}$ of its position, although experience with high resolution spectra of microlensed bulge main sequence stars indicates that the extinction toward the source is not likely to differ from the average extinction toward the neighboring red clump stars by more than 5\% (Cohen et al. 2009). With measurements of the source star brightness in the three VI H passbands, we can make use of the Kenyon \& Hartmann (1995) color-color relations for dwarf stars to demand that the extinction is consistent with the colors of a main sequence star, as well as approximately matching the extinction law and value derived for the red clump stars. We use

$$
H_{s}=18.876 \pm 0.030
$$


Table 1

Nonlinear Model Parameters

\begin{tabular}{lccc}
\hline \hline Parameter & Units & Best-fit Value & MCMC Range \\
\hline$t_{E}$ & Days & 127.300 & $128.1 \pm 0.8$ \\
$t_{0}$ & HJD $-2,450,000$ & 3831.0197 & $3831.0204 \pm 0.011$ \\
$u_{0}$ & & 0.003479 & $0.00345 \pm 0.00005$ \\
$d_{1 \mathrm{~cm}}$ & 0.6272 & $0.632 \pm 0.073$ \\
$d_{23}$ & & 1.04185 & $1.0418 \pm 0.0001$ \\
$\theta_{1 \mathrm{~cm}}$ & Radians & 2.52297 & $2.5232 \pm 0.0007$ \\
$\phi_{23}$ & Radians & -0.23560 & $-0.2350 \pm 0.0007$ \\
$\epsilon_{1}$ & & $1.3562 \times 10^{-3}$ & $(1.350 \pm 0.013) \times 10^{-3}$ \\
$\epsilon_{2}$ & & $5.0516 \times 10^{-4}$ & $(5.017 \pm 0.030) \times 10^{-4}$ \\
$t_{*}$ & & 0.03972 & $0.03949 \pm 0.00016$ \\
$\dot{d}_{23 x}$ & Days & 0.00169 & $0.00171 \pm 0.00004$ \\
$\dot{d}_{23 y}$ & Days & $0.00179 \pm 0.00014$ \\
$1 / T_{\text {orb }}$ & Days & 0.00181 & $2.3 \pm 0.7 \times 10^{-4}$ \\
$\pi_{E}$ & yr $^{-1}$ & $2.04 \times 10^{-4}$ & $0.345 \pm 0.014$ \\
$\phi_{E}$ & & 0.3620 & $2.728 \pm 0.010$ \\
Fit $\chi^{2}$ & Radians $^{-1}$ & 2.7296 & \\
\hline
\end{tabular}

Notes. Static parameters describe configuration at $\mathrm{HJD}-2,450,000=3831.0$ (i.e., close to $t_{0}$ ). Mass 1 refers to the (Jupiter-mass) planet $b$, mass 2 is the (Saturn-mass) planet $c$, and mass 3 is the host star.

$$
\begin{aligned}
& I_{s}=20.935 \pm 0.030, \\
& V_{s}=23.110 \pm 0.030
\end{aligned}
$$

for the magnitudes of the source star. Note that we deliberately overestimate the error bars in Equations (9)-(11) to account for the uncertainties in the color-color relations. We also require that the Cardelli et al. (1989) extinction parameter, $R_{v}$, matches the value determined for the red clump stars within $1^{\prime}$ of the source and that the total $H$-band extinction be within $5 \%$ of the value determined for the red clump stars. Using the magnitudes in Equations (9)-(11) and the derived extinction estimates, we then use the $(V-H, H)$ color-radius relation of Kervella et al. (2004) to derive the angular radius of the source star.

We use two methods to impose these constraints and determine the source radius. The first method is to impose these constraints using an MCMC calculation that selects star colors at random with the requirement that they obey the Kenyon \& Hartmann (1995) color-color relations and $R_{v}$ and $A_{H}$ selected from the probability distributions mentioned above. The $\chi^{2}$ is calculated for the $V-H, V-I$, and $I-H$ colors derived from Equations (9)-(11). This $\chi^{2}$ is multiplied by $2 / 3$ before being used in the Metropolis et al. (1953) algorithm to account for the fact that these colors satisfy $V-H=(V-I)-(I-H)$. Since each point on the Markov Chain gives unique values for the dereddened source magnitude, $H_{s 0}$ and color, $(V-H)_{s 0}$, each point on the chain specifies a unique $\theta_{*}$ value.

Our second method is to consider all combinations of $R_{v}$ and source color (subject to the color-color relations), and then adjust $A_{H}$ to minimize the $\chi^{2}$ with the colors constrained by the color-color relations. This results in the $\chi^{2}$ versus $\theta_{*}$ distribution shown in Figure 8, which is approximately bounded by the $\theta_{*}=0.468 \pm 0.012 \mu$ as curve derived from the MCMC calculation. However, this estimate does not include the quoted $1.1 \%$ uncertainty in the Kervella et al. (2004) color-radius relation. When this is added, we find

$$
\theta_{*}=0.468 \pm 0.013 \mu \mathrm{as}
$$

for the angular radius of the source star. This can be combined with two light-curve parameters, the Einstein radius crossing

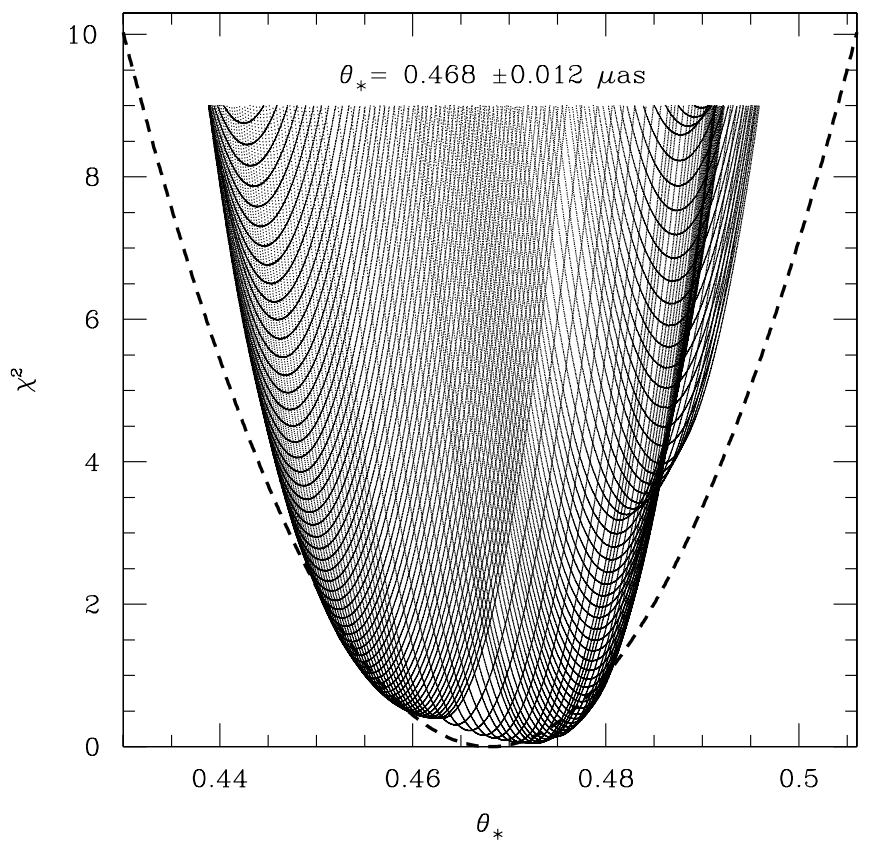

Figure 8. $\chi^{2}$ vs. angular source radius, $\theta_{*}$, is plotted for stars obeying the color-color relations of Kenyon \& Hartmann (1995) subject to extinction following the Cardelli et al. (1989) model using the $R_{v}$ distribution found for the red clump stars within $1^{\prime}$ of OGLE-2006-BLG-109S. The dashed curve is the $\chi^{2}$ parabola describing the result of our MCMC calculation: $\theta_{*}=0.468 \pm 0.012 \mu$ as. The $H$-band extinction is assumed to be within $5 \%$ of the $A_{H c}=0.60$ value derived for this set of red clump stars.

time, $t_{E}$, and the source radius crossing time, $t_{*}$, to yield the angular Einstein radius, $\theta_{E}=\theta_{*} t_{E} / t_{*}=1.505$ mas, using the best-fit parameters listed in Table 1 . We do not include error bars here because the complete analysis of the uncertainties is given in Section 9.

With this determination of $\theta_{E}$, we can use the length of the microlensing parallax vector to determine the lens mass,

$$
M_{L}=\frac{\theta_{E} \tilde{r}_{E} c^{2}}{4 G}=\frac{\theta_{E} c^{2} \mathrm{AU}}{4 G \pi_{E}}=\frac{\theta_{E} M_{\odot}}{(8.1439 \mathrm{mas}) \pi_{E}} .
$$

Using the best-fit value of $\pi_{E}=0.3619$ from Table 1 this gives $M_{L}=0.51 M_{\odot}$. Since the distance to the source star is 
generally known, at least approximately, we can also determine the distance to the lens star:

$$
D_{L}=\frac{D_{S}}{1+\frac{\pi_{E} \theta_{E} D_{S}}{1 \mathrm{AU}}} .
$$

If we assume $D_{S}=8.0 \mathrm{kpc}$ and the fit parameters from Table 1, this yields $D_{L}=1.49 \mathrm{kpc}$. Note that for our adopted parameters, the fractional error in $D_{L}$ induced by the error in $D_{S}$ is a factor of 0.19 smaller than the fractional error in $D_{S}$ itself, so the uncertainty in $D_{L}$ is quite small.

\section{PHYSICAL CONSTRAINTS ON MODEL PARAMETERS}

In principle, it is possible for a planet detected in a microlensing event to be physically unrelated to the lens star, but this is quite unlikely. If the signal occurs near the peak of a high magnification event, the probability is extremely small, $\lesssim 10^{-8}$ per event. So, with less than 100 high-magnification microlensing events observed, the probability that an unrelated planet has been seen is $<10^{-6}$. (Furthermore, if two lens objects are closely aligned by chance, they will generally be at different distances, so the usual binary lens magnification equations do not apply (Rhie \& Bennett 2009).) So, we will assume that the planets detected in the OGLE-2006-BLG-109 light curve are in orbit about the lens star, OGLE-2006-BLG-109S. With this assumption, we can use the lens mass determined from Equation (13) to constrain the parameters describing the orbital motion: the velocity in the plane of the sky, described by $\dot{d}_{23 x}$ and $\dot{d}_{23 y}$, and the orbital period, $T_{\text {orb }}$.

When the mass of the lens system is unknown, there are seven parameters needed to describe the relative orbital motion of two masses. (These can be taken to be the initial relative position and velocity, plus the reduced mass that is needed for the gravitational equation of motion.) Since we know the lens mass from Equation (13), it might seem that we can reduce the number of free parameters to six, but there is a complication. The lens separation and relative velocity parameters are given in units of the (linear) Einstein ring radius, $R_{E}$, but the gravitational equation of motion requires standard physical length units. So, in order to make use of the measured lens system mass to reduce the number of parameters, we need to know the linear Einstein radius $R_{E}=D_{L} \theta_{E}$. Although we can use Equation (14) to give us the lens distance, $D_{S}$ is only approximately known, and therefore this cannot give a precise constraint on the lens parameters.

We will consider two sets of constraints, corresponding to two different interpretations of our set of orbital parameters. As mentioned in Section 3, our models have the three parameters describing orbital motion and two parameters giving the instantaneous position of the second planet $\left(d_{23}\right.$ and $\left.\phi_{23}\right)$, so our models have five parameters to describe the orbits. As discussed in Section 3, these parameters are sufficient to characterize a circular orbit, which is what our lensing model assumes. If we restrict our consideration to circular orbits, then we can use Equations (13) and (14) to constrain the model parameters. However, since $D_{S}$ is known only approximately, we do not impose a hard constraint on the fit parameters. Instead, we invert Equations (13) and (14) to find an expression for $D_{S}$. We then add a term to $\chi^{2}$ of the form

$$
\chi_{D_{S}}^{2}=\left(D_{S}-D_{S 0}\right)^{2} / \sigma_{D_{S}}^{2}
$$

to impose a "soft" constraint on the lens parameters during the modeling process. The models we present here used
$D_{S 0}=8.0 \mathrm{kpc}$ and $\sigma_{D_{S}}=1.5 \mathrm{kpc}$. This $\sigma_{D_{S}}$ is a little larger than the prediction of most bulge models, but we pick a conservative value as we do not need a tight circular orbit constraint.

The advantage of this constrained circular orbit modeling scheme is that it prevents the modeling runs from straying into the wide swaths of parameter space that do not correspond to physical orbits. Also, for this event, the orbital period parameter, $T_{\text {orb }}$, is only very weakly constrained, so there is little danger that this constraint will prevent the modeling and MCMC runs from reaching the vicinity of the correct model in parameter space. If $T_{\text {orb }}$ were more tightly constrained, then it might be sensible to try a weaker constraint on $D_{S}$ that just ensures that the fit velocity is not large enough to make the total energy positive, implying an unbound system.

While the constrained circular orbit scheme is a useful way to explore parameter space to ensure that all the viable models are considered, it cannot be used to work out the constraints on the physical orbital parameters of the system. When we consider this question in Sections 8 and 9, we use a different interpretation of the $T_{\text {orb }}$ parameter. Formally, $T_{\text {orb }}$ is the period of the circular orbit of the planet. However, if we expand the motion of the Saturn-mass planetary lens in a Taylor series, we will see that there is an alternative interpretation. The first-order correction to the static two-dimensional Saturn-mass planet position used for most events is the two-dimensional velocity of this planet, which is strongly constrained by the photometric data for OGLE-2006-BLG-109. However, the direction of the next order acceleration term is constrained by Newton's law of gravity to be toward the stellar lens mass. Since our orbit model has only a single additional parameter beyond the firstorder velocity parameters, this additional parameter, $T_{\text {orb }}$, must be equivalent to the orbital acceleration to second order. And since $T_{\text {orb }}$ is weakly constrained by the data, we can be confident that the higher order terms are essentially unconstrained by the data. This allows us to make an alternative interpretation of the $T_{\text {orb }}$ parameter for this event. We can interpret it as the orbital acceleration. This interpretation is used in Sections 8 and 9, where we derive constraints on the orbital parameters of OGLE-2006-BLG-109Lc (the Saturn-mass planet).

\section{FOLLOW-UP OBSERVATIONS AND ANALYSIS}

We have obtained follow-up adaptive optics (AO) images from the Keck Observatory and spectra from the Magellan Telescope in an attempt to characterize the planetary host star and the neighboring bright red clump star. The Keck observations and interpretation are discussed in Sections 6.1-6.3, and the Magellan spectra are discussed in Section 6.4.

\subsection{Keck Observations}

The close-up of the OGLE finding chart shown in the lefthand panel of Figure 9 indicates that the event appears to be centered on a relatively bright star. This is the red clump giant star indicated by the green dots in the CMDs shown in Figures 5-7. These CMDs also indicate that the source is some 4.1-4.6 mag fainter than this red clump star (depending on the passband), so this bright star is obviously not the source. We initially considered the possibility that this could be the lens star, which would have been inconsistent with the photometric properties implied by the lens mass and distance implied by the microlensing parallax and $\theta_{E}$ measurement as discussed in Section 4. However, an astrometric analysis of the OGLE data indicated that the lensing event is centered 0.31 north of 


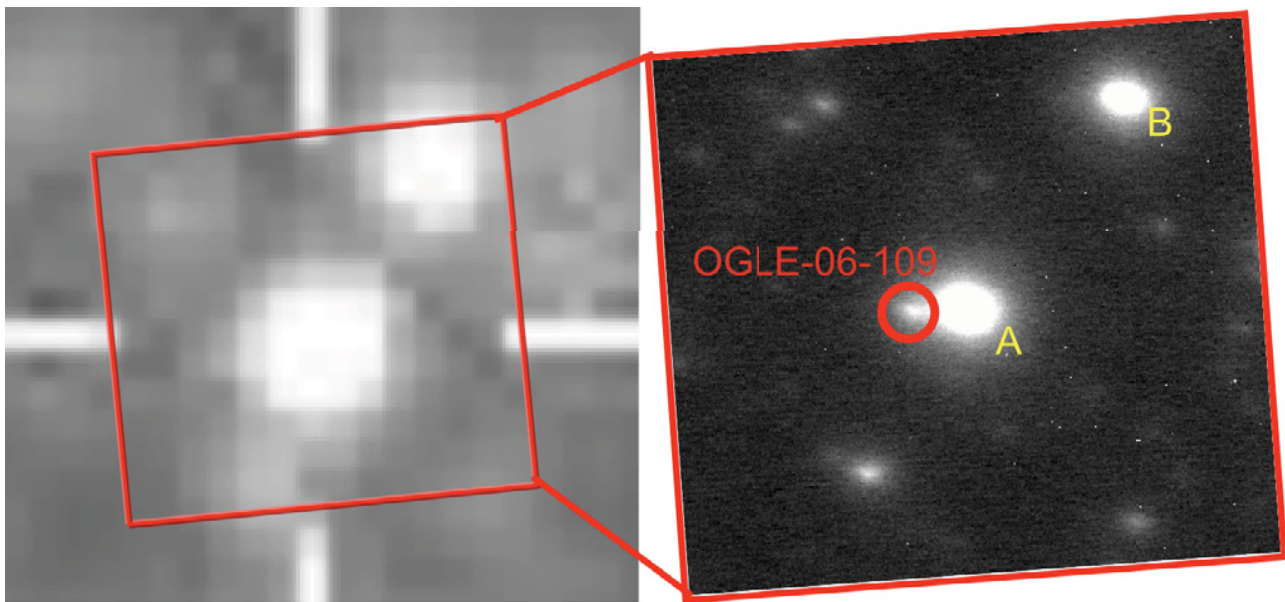

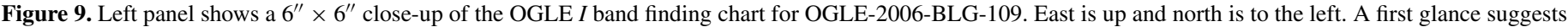

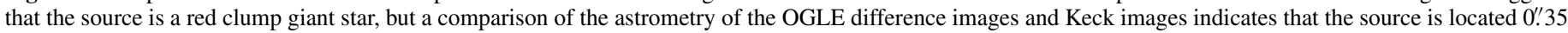

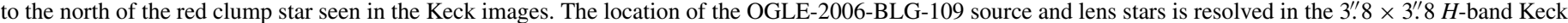
AO image on the right.

the centroid of the relatively bright object at the center of the crosshairs in the left panel of Figure 9. This means that most of the flux in this star-like image is due to a star that is unrelated to the microlensing event. (We show that this star does not produce any significant lensing effect in Section 10.)

In 2007 July, we obtained $H$ - and $K$-band AO images of OGLE-2006-BLG-109 with the NIRC2 instrument on the Keck2 telescope. The $\mathrm{AO}$ correction was made using a natural guide star located $38^{\prime \prime}$ from the target. As a result of this relatively large angular distance to the guide star, the $\mathrm{AO}$ correction is not as good as the Keck AO system normally provides. The image FWHM values range from $00^{\prime} 09$ to $0{ }^{\prime} .13$ in the $K$ band and from 0.15 to $0^{\prime} .25$ in the $H$ band. The right-hand panel of Figure 9 shows the best seeing $H$-band image. While this is not a very good correction by Keck standards, it is fine for our main purpose which is to resolve the combined image of the lens and source stars (in the red circle in Figure 9) from this much brighter, unrelated star (labeled A in Figure 9).

Although the $K$-band images have better image quality, we focus on the $H$-band data because we have $H$-band data from CTIO during the event that allows us to determine the brightness of the source star in the $H$ band. The reduction of the Keck AO data requires a crowded field photometry code because of the significant overlap in the images of our target star with the bright red clump star, labeled " $\mathrm{A}$ " in Figure 9. The reduction is done with DAOPHOT (Stetson 1994) using the "penny1" PSF function with a 62 pixel radius. (The pixels subtend 0.'01.)

The PSF shape changes significantly across the AO images, perhaps as a result of the relatively large $38^{\prime \prime}$ angular distance to the guide star and the high airmass of the Galactic bulge as observed from Hawaii. In order to avoid significant photometry errors due to the spatial dependence of the PSF, we only use two relatively bright stars within $3^{\prime \prime}$ to do the relative calibration of the Keck $H$-band photometry to CTIO. These stars are labeled A and B in Figure 9. The CTIO photometry is then calibrated to the 2MASS system (Skrutskie et al. 2006) using 16 stars in common between CTIO and 2MASS that have been specifically selected to be uncrowded in the CTIO frames to avoid systematic errors due to blending in the relatively poor seeing 2MASS images.

This analysis yields a total lens plus source magnitude of $H_{l s}=16.99 \pm 0.04$, which can be combined with the source magnitude of $H_{s}=18.876 \pm 0.014$ from the light- curve model to yield a lens magnitude of $H_{L}=17.17 \pm$ 0.05 . The contributions to this final uncertainty in $H_{l}$ are $1 \%$ from the CTIO-2MASS calibration, 2\% from the CTIO-Keck calibration, and $4 \%$ from the Keck photometry.

We can now compare this magnitude to the expectations based on our calculations in Section 4. Using the mass-luminosity relations of Kroupa \& Tout (1997), we predict an absolute $H$ magnitude of 5.94 for the $M_{L}=0.51 M_{\odot}$ lens star. At a distance of $D_{L}=1.49 \mathrm{kpc}$, this yields a dereddened lens magnitude of $H_{L 0}=16.81$. However, the "best-fit" model considered in Section 4 actually included an $H$-band magnitude constraint, so to avoid circular reasoning, we should actually use the best-fit model without such a constraint. This model is quite similar, but predicts $M_{L}=0.52 M_{\odot}$ and $D_{L}=1.53 \mathrm{kpc}$, which would imply an absolute $H$ magnitude of 5.87, and $H_{L 0}=16.79$. Now, the extinction in the foreground of the source is $A_{H}=0.60 \mathrm{mag}$, but the source is located at Galactic coordinates of $l=-0.2086$ and $b=-1.8901$, which means that the lens system is about $35 \mathrm{pc}$ south of the Galactic plane. As a result, it is likely that there is a significant amount of dust in the foreground of the lens system and between the lens and source. So, we might guess that the extinction toward the lens is $A_{H L} \approx 0.3 \pm 0.2$. This leads to the prediction of $H_{L}=17.09 \pm 0.20$, which of course, matches our Keck measurement quite well.

\subsection{Must the Excess H-Band Flux Seen by Keck Be from the Lens Star?}

Before ending our discussion of the Keck observations, we should also consider the possibility that another star besides the planet host star is responsible for this detected $H$-band flux. There have now been several events that have also had the detection of an additional star superimposed on top of the source in a high resolution image. For the first two planets found by microlensing, images with the Hubble Space Telescope (HST) indicated different centroid positions for the source plus blend star in different colors, which was consistent with the expected offset due to the lens-source proper motion determined from the light curve (Bennett et al. 2006; Dong et al. 2009a). So, for these events, it is quite likely that the additional flux is due to the planetary host/lens star. However, for MOA-2008-BLG310 , where excess flux that could in principle be associated with 
the lens was also detected, the light-curve parameters imply that the lens must be only $\sim 300 \mathrm{pc}$ from the source if it is responsible for this excess flux. The a priori probability of such a small lens source distance is comparable to the a priori probability that the excess flux is due to a binary companion to the source or lens star, or even a completely unrelated star. So, this event is ambiguous (Janczak et al. 2010)

The situation is quite different for OGLE-2006-BLG-109. In this case, it is very unlikely that the excess flux superimposed on the source is due to a star other than the lens/planetary host star for a couple of different reasons. First, the excess flux in $H$ matches the prediction for the mass and distance of lens star as determined from the microlens parallax and finite source effects, and second, it is brighter than bulge main sequence stars, the number of stars with the observed brightness is relatively low. Nevertheless, it is a logical possibility that the planet host could be a dim white dwarf and the detected flux could be from another star, although we note that despite several systematic searches (Mullally et al. 2008; Hogan et al. 2009; Kilic et al. 2009), the only planet known to orbit a white dwarf was apparently involved in a complicated dynamical interaction in the core of a globular cluster (Sigurdsson et al. 2003). We now proceed to estimate the probability that another star besides the planetary host star could be responsible for the detected $\mathrm{H}$ band flux using arguments similar to those of Janczak et al. (2010). There are three possibilities: the chance superposition of an unrelated star or a binary companion to the lens or source star.

The most likely alternative is the chance superposition of an unrelated star. The density of stars within 0.4 mag of the $H$-band flux attributed to the lens star is 0.126 stars per square arcsecond, so the probability that such a star is close enough to the source so that the source is not separately detected in the best seeing $K$-band image is $0.3 \%$ assuming that the star can just be detected at a separation of 1 FWHM.

The next possibility is that the excess $H$-band flux could be from a binary companion to the source star. Unlike the case of MOA-2008-BLG-310 (Janczak et al. 2010), the companion would have to be substantially brighter than the source. It would be 1.7 mag brighter than the source in the $H$ band, which would make it brighter than the top of the bulge main sequence (Holtzman et al. 1998; Zoccali et al. 2000). With the same extinction and distance as the source, the absolute $H$-band magnitude of this companion would be $\sim 2.05$, which would imply that it should be a hydrogen-shell burning star just beginning its rise up the red-giant branch. Assuming a typical bulge age of $\sim 10 \mathrm{Gyr}$, it would have a mass of just over a solar mass and about $10 \%-15 \%$ more massive than the source. Figure 10 of Duquennoy \& Mayor (1991) implies that about $1.4 \%$ of $\mathrm{G}$ dwarfs should have a binary companion that is 1-1.1 times more massive. However, this phase of stellar evolution is quite short-lived. From Sackmann et al. (1993), we estimate that the Sun will have an absolute $H$ magnitude of $2.05 \pm 0.3$ for a time period equal to only about $2 \%$ of its main sequence lifetime. Furthermore, a star's lifetime varies roughly as the -2.5 power of the mass, so the initial mass interval corresponding to bulge stars with an absolute $H$-band magnitude of $2.05 \pm 0.3$ is only $0.08 M_{\odot}$. This means that only $8 \%$ of the bulge G-dwarfs with companions in the $1-1.1 M_{\odot}$ mass range should be in this magnitude range. Finally, based on Figure 7 of Duquennoy \& Mayor (1991), we expect that $25 \%$ of these binary companions would have a separation of $>700 \mathrm{AU}$ where they could be detected in the best Keck images, and another
$17 \%$ would have an orbital period of $<200$ days which would imply a detectable "xallarap" (source orbital motion) effect in the microlensing light curve. So, the a priori probability of a source companion with the observed $H$-band magnitude is only $0.06 \%$

The final alternative is a companion to the lens. This possibility is excluded by the lack of any signature of an additional companion in the light curve. In this scenario, the companion must be at the same distance as the lens, so the $H$-band brightness means that lens must be a white dwarf of $\sim 0.5 M_{\odot}$, and the companion must be an M-dwarf of essentially the same mass. The light curve shows no evidence of such a companion, so we use the method of Rhie et al. (2000) to work out the constraints on an additional lens companion at the same mass as the lens star, and this analysis indicates that such companions separated by less than 0 '. 18 from the primary lens star are excluded. However, the lens and source should be separately detected in the best $K$-band image if the separation is $>0 . ' 09$, so this possibility is ruled out.

So, the a priori probability that source of the excess flux observed in the Keck AO images is something other than the lens star is less than $0.4 \%$ under the assumption that white dwarfs are as likely to host planets as early-M dwarfs.

\subsection{Lens Brightness Constraint}

Now that we have shown that it is extremely unlikely for the excess $H$-band flux superimposed on the source star to belong to another star, beside the lens star, we will make the assumption that the flux we detect is from the lens star. Our measurement gives $H_{L}=17.17 \pm 0.05$, but at its likely distance of $D_{L} \approx 1.5 \mathrm{kpc}$, the lens is only located about $35 \mathrm{pc}$ from the Galactic plane, and so the extinction toward the lens is likely to be substantially less than the extinction toward the source. This means that we cannot use all the Galactic bulge stars within a small angular distance from the lens star to estimate the foreground extinction.

The distribution of the dust in the Galactic disk is somewhat complicated (Drimmel \& Spergel 2001; Marshall et al. 2006), and the distribution along the line of sight at a distance of $D_{L} \approx 1.5 \mathrm{kpc}$ toward the Galactic center is not very well known. So, we will use a very simple model, with generous error bars. At $1.0 \mathrm{kpc}$ interior to the solar circle, the scale height of the dust distribution is about $110 \mathrm{pc}$. At a Galactic latitude of $b=-1.8901$, the decrease in dust density due to the line of sight leaving the disk plane is essentially canceled by the increase in density due to the approach to the Galactic center. However, some models have a flared disk that decreases the scale height at smaller Galactocentric radii. Also, the dust distribution is not thought to continue all the way into the Galactic bulge. To get a crude estimate of the extinction, we simply assume a scale height of $110 \mathrm{pc}$ that continues all the way to the source. For source extinction of $A_{H S}=0.60$, this gives $A_{H L}=0.24 \pm 0.24$, where we have assumed a large uncertainty because of the crudeness of this estimate. Of course, we do not allow $A_{H L}<0$, and $A_{H L}=0$ should be quite unlikely. But, at these coordinates, some of the dust models imply that the dust density increases all the way to the bulge. So, it is quite possible that only a very small fraction of the extinction is in the foreground of the lens star. Combining this with the estimated lens star $H$-band brightness, we find a dereddened lens star brightness of

$$
H_{L 0}=16.93 \pm 0.25 \text {, }
$$


which we can use to compare to the predictions of the lensing models.

\subsection{Magellan Spectrum and Host Star Kinematics}

A spectrum of the lens star, the source star, and the bright star 0.35 to the north of the lens and source was obtained using the MIKE Spectrum on the Magellan Telescope by G. Pietrzyński on 2007 March 25. This spectrum covered the spectral range 5400-6500 ̊. It indicates that this bright neighboring star is indeed a red clump giant star as its positions in the CMDs imply. (See Figures 5-7.) A cross-correlation analysis of this spectrum was kindly provided by Ian Thompson and Andy McWilliam of the Carnegie Observatories, using HD193901 (a metal poor subgiant) as the template. This cross-correlation analysis was done with the IRAF FXCOR package, and used the spectral ranges 5400-5875 $\AA$ and 5925-6500 $\mathrm{to} \mathrm{avoid} \mathrm{strong}$ interstellar absorption in the $\mathrm{Na}-\mathrm{D}$ lines. The resulting crosscorrelation function clearly shows two peaks. The strongest peak has a Heliocentric radial velocity of $v_{r}=125 \mathrm{~km} \mathrm{~s}^{-1}$, and the second strong peak has a Heliocentric radial velocity of $v_{r}=-49 \mathrm{~km} \mathrm{~s}^{-1}$. The peak ratio was about 2.6, and the formal uncertainties reported by the IRAF FXCOR package was less than $1 \mathrm{~km} \mathrm{~s}^{-1}$ for each peak. We attribute the strongest peak to the bright blend star and the second strongest peak to the planetary host star. The source star is too faint to be detected by this analysis.

The radial velocity of the blend star is consistent with our classification of it as a bulge giant, but it is not otherwise interesting. The kinematics of the host star can be compared to those of some 528 stars with $M_{V}>4$ within $25 \mathrm{pc}$ of the Sun as compiled by Reid et al. (2002). Converted from the Heliocentric frame to one at rest with the average of this nearby star population, we find that the radial velocity of the host star is $v_{r}=-36 \mathrm{~km} \mathrm{~s}^{-1}$, which has an absolute value slightly smaller than the rms value of $40 \mathrm{~km} \mathrm{~s}^{-1}$.

We do not have a direct measure of the transverse motion of the host star, but the microlensing parallax and finite source measurement does give us the relative velocity between the host and source stars. Since the distance to the host, $D_{L} \approx 1.5 \mathrm{kpc}$, is much smaller than the distance to the source, $D_{S}$, this tells us mostly about the host star. If we assume that the source is a bulge star, and the bulge has no rotation and has a velocity dispersion of $80 \mathrm{~km} \mathrm{~s}^{-1}$ in both the Galactic North and rotation directions, then we find that the velocity distribution that we infer for the host star is $\left(v_{V}, v_{W}\right)=(-45,-19) \pm(15,15) \mathrm{km} \mathrm{s}^{-1}$, where $v_{W}$ and $v_{V}$ are the components of the lens velocity in the Galactic North and rotation directions. Here, the uncertainty is entirely due to the bulge velocity dispersion. The velocity dispersion for the local star sample (Reid et al. 2002) is $\left(\sigma_{W}, \sigma_{V}\right)=(28,19) \mathrm{km} \mathrm{s}^{-1}$. We can add this in quadrature to the uncertainty due to the unknown source star velocity, and we find $\left(v_{V}, v_{W}\right)=(-45,-19) \pm(32,24) \mathrm{km} \mathrm{s}^{-1}$. So, two of three components of the host star velocity are within $1 \sigma$ of the expected value and the third component is $1.4 \sigma$ behind the mean velocity in the rotation direction. So, the kinematics are quite consistent with a bulge source and a relatively old planetary host star in the disk, as we would expect.

\section{ALTERNATIVE LENS MODELS}

High-magnification planetary microlensing events are known to have a number of approximate parameter degeneracies that can often complicate the interpretations of these events. We
Table 2

Physical Parameters

\begin{tabular}{lcccccc}
\hline \hline Parameter & Units & \multicolumn{5}{c}{ Parameter Limits } \\
\cline { 3 - 7 } & & $-2 \sigma$ & $-1 \sigma$ & Median & $+1 \sigma$ & $+2 \sigma$ \\
\hline$D_{L}$ & $\mathrm{kpc}$ & 1.30 & 1.39 & 1.51 & 1.62 & 1.74 \\
$M_{A}$ & $M_{\odot}$ & 0.43 & 0.47 & 0.51 & 056 & 0.60 \\
$m_{b}$ & $M_{\oplus}$ & 195 & 212 & 231 & 250 & 268 \\
$m_{c}$ & $M_{\oplus}$ & 73 & 79 & 86 & 93 & 99 \\
$M_{\mathrm{H}}$ & & 5.45 & 5.68 & 5.90 & 6.13 & 6.33 \\
$a_{b}$ & $\mathrm{AU}$ & 1.6 & 1.8 & 2.3 & 2.8 & 3.4 \\
$P_{b}$ & Years & 2.8 & 3.4 & 4.9 & 6.5 & 7.3 \\
$a_{c}$ & $\mathrm{AU}$ & 2.9 & 3.5 & 4.5 & 6.6 & 13.5 \\
$P_{c}$ & Years & 6.7 & 8.7 & 13.5 & 23.2 & 68 \\
$\epsilon_{c}$ & & 0.007 & 0.05 & 0.15 & 0.32 & 0.62 \\
$\alpha_{c}$ & $\mathrm{deg}$ & -50 & -43 & -36 & -26 & -16 \\
$i_{c}$ & $\mathrm{deg}$ & 49 & 56 & 64 & 68 & 73 \\
$K_{b}$ & $\mathrm{~m} \mathrm{~s}^{-1}$ & 14.6 & 16.3 & 17.4 & 18.7 & 19.9 \\
$K_{c}$ & $\mathrm{~m} \mathrm{~s}$ & 2.8 & 3.9 & 4.5 & 5.0 & 5.3 \\
\hline
\end{tabular}

Notes. $M_{H}$ is the absolute $H$-band magnitude of the planetary host star.

explore these alternative models with respect to the reference model with $\chi^{2}=2542.06$ for 2557 degrees of freedom. (See Table 2 for the parameters of this model.) The first of these degeneracies is the well known $d \leftrightarrow 1 / d$ degeneracy (Griest \& Safizadeh 1998; Dominik 1999) that applies to high magnification events unless $d \approx 1$. In the case of OGLE-2006BLG-109, this would apply to the Jupiter-mass planet at $d \approx 0.6$ (or $d \approx 1.6$ ), but not to the Saturn-mass planet at $d=1.04$. This Saturn-mass planet is a case of a so-called resonant caustic, in which the planetary caustic is connected with the central caustic. Since the source trajectory encounters the planetary part of the caustic, the $d \leftrightarrow 1 / d$ degeneracy is broken for this planet. In Gaudi et al. (2008), we reported that the degenerate solution with $d_{J} \approx 1.6$ was disfavored by $\Delta \chi^{2}=11.4$, which was enough for us to formally exclude this solution. However, the situation is a bit more complicated in the current analysis, as we describe below.

The other approximate degeneracies concern the microlensing parallax effect. These have been discussed in the context of single lens events by Smith et al. (2003), Gould (2004), and Poindexter et al. (2005). The symmetries of the first few terms of the Taylor expansion of the parallax effect (with respect to time) lead to approximate degeneracies of the full effect for most events, which have $t_{E} \ll 1 \mathrm{yr}$. However, one of the symmetries of single- and double-lens models is removed by the third lens. High-magnification planetary microlensing events often have four degenerate parallax solutions (Bennett et al. 2008), but an additional planetary mass ruins the reflection symmetry about the lens axis (because there is no longer a unique lens axis). As a result, there is only a single discrete degeneracy that remains.

With the modeling runs used for the OGLE-2006-BLG-109 discovery paper, there was a degenerate model with parameters similar to those listed in Table 1 except that $u_{0}=-0.00344$, $\theta_{1 \mathrm{~cm}}=-2.5266, \phi_{E}=0.684$, and $\pi_{E}=0.239$. This model was disfavored compared to the best-fit model presented in that paper by $\Delta \chi^{2}=7.6$. Although this $\Delta \chi^{2}$ alone is formally enough to exclude this model, it was further disfavored because the change in $\pi_{E}$ value implies a much larger lens system mass of $M_{L} \approx 0.77 M_{\odot}$ at a somewhat larger distance of $D_{L}=2.06 \mathrm{kpc}$, which would predict an $H$-band brightness that is $\sim 0.8$ mag brighter than the observed $H$-band magnitude, which corroborates the rejection of this model. 
The rejection of this alternate model is further confirmed by our inclusion of the terrestrial parallax effect in our current modeling. This is simply the parallax effect due to the locations of the different observatories on the surface of the Earth. In our previous modeling, we effectively assumed that all observations take place from the center of the Earth. With the inclusion of this effect, the best-fit $\chi^{2}$ improves by $\Delta \chi^{2}=13.5$, but the $\chi^{2}$ difference with the $u_{0}<0, \theta_{1 \mathrm{~cm}} \approx-2.5, \phi_{E} \sim 0.7$ model increases to $\Delta \chi^{2}=39.3$. So, we can consider this model to be strongly excluded by several lines of evidence.

However, the situation is more complicated with the $d_{J}>1$ model. When terrestrial parallax is added to this model, the $\chi^{2}$ of this model improves by more than the $d_{J}<1$ model, so that the $\chi^{2}$ difference between these models drops to $\Delta \chi^{2}=1.8$. Thus, the model with the Jupiter orbiting outside the Saturn is no longer formally excluded by this $\chi^{2}$ difference. However, as we shall discuss in Section 8, this best-fit $d_{J}>1$ model is almost completely inconsistent with any co-planar stable orbit, so the conclusion of Gaudi et al. (2008) that the Jupiter orbits inside the Saturn is still likely to be correct.

\section{CONVERTING MODEL PARAMETERS TO PHYSICAL ORBITS}

As we mentioned in Section 5, there are two possible interpretations of our orbital motion modeling scheme. The first interpretation is to take the circular orbit model literally. This is an effective way to ensure that the orbital model parameters do not stray into unphysical regions of parameter space. But, of course, it is unlikely that the orbit of the Saturn-mass planet is precisely circular, but our orbital model allows only a circular orbit.

In order to extract the set of physical orbits that are consistent with the parameters of a given model, we adopt the second interpretation of our orbital parameter models. In this interpretation, the $1 / T_{\text {orb }}$ parameter is considered to be a measure of the orbital acceleration, to which it is equivalent at second order. With this interpretation, we compute the acceleration implied by the circular orbit model. Then, using the measured $\theta_{*}$ value and Equations (13) and (14) we can determine the relative distance along the line of sight between the Saturn-mass planet and its host star. With the other fit parameters, this gives us the three-dimensional position and the velocity in the plane of the sky. Since the mass is known from Equation (13), this leaves one additional parameter, the velocity along the line of sight, to describe the orbit. Thus, we have a single parameter family of orbits for each set of model parameters. Given a set of fit parameters, we first calculate the minimum energy of the orbit using the three-dimensional position of the planet and the two-dimensional velocity. If this energy is positive, then there are no bound orbits consistent with these fit parameters. If the energy is negative, then we can construct the family of orbital solutions by varying the velocity along the line of sight from the maximum value consistent with a bound orbit to the negative of that value.

It may seem odd that we can use a model of describing a circular orbit that can be interpreted to describe a one-parameter family of general orbits, because it normally requires two more parameters to describe an orbit that is not constrained to be circular. However, if we assume that the distance to the source is known, the circular orbit problem is over-constrained by model parameters, the application of Kepler's third law and the measurement of the source star angular radius, $\theta_{*}$. We avoid this problem of too many constraints, by considering $D_{S}$ to be a variable, with the soft constraint, Equation (15). Now, when we generalize to non-circular orbits, we fix $D_{S}$ and use the additional constraint to avoid an additional non-circular orbit parameter.

The duration of the light-curve signal of the Jupiter-mass planet is too brief to reveal any information regarding its orbit. However, it is well accepted that planet formation occurs in a disk, so that all planets are expected to form in one plane. Furthermore, the only known exoplanet systems that have measured inclinations of more than one planet are the solar system and the two most massive planets of the PSR 1257+12 system (Konacki \& Wolszczan 2003). In both cases the planets orbit in nearly the same plane. Thus, it is natural to assume that the planets in the OGLE-2006-BLG-109L system are also co-planar. If so, we can use this assumption to provide tighter constraints on the orbital parameters of the Saturn-mass planet, OGLE-2006-BLG-109Lc.

In addition to requiring that the Saturn-mass planet has parameters that correspond to a bound orbit, we can also require that its orbit and that of the Jupiter-mass planet are in a stable configuration. With the assumption that the orbits are co-planar, we can define a unique three-dimensional position for the Jupiter, once the orbit of the Saturn-mass planet has been defined. As described above, this requires that we specify a line-of-sight velocity for the Saturn-mass planet in addition to the parameters that describe the light-curve model. With only the position of the Jupiter-mass planet specified, we have a lot of freedom for the rest of the orbital parameters. However, the choice that will impose the weakest orbital stability constraints on the orbit of the Saturn-mass planet is simply to assume a circular orbit for the Jupiter-mass planet, so this is what we assume.

With the orbits of both planets now specified, we can now check for orbital stability. The simplest constraint is to insist that the orbits do not cross, but orbits do not have to cross to be unstable. We employ the analytic Hill stability criterion of Barnes \& Greenberg (2006) as our condition for orbital stability. While the majority of unstable orbits are removed by the simple orbit crossing criterion, it is only the Hill stability criterion that can detect unstable circular orbits, and it is in fact this latter condition that excludes the best-fit $d_{J}>1$ model discussed in Section 7. In particular, only $0.04 \%$ of the parameter sets in our MCMC chain calculations which corresponded to stable orbits with $d_{J}>1$. However, some of the $d_{J}<1$ Markov chains were much longer than the $d_{J}>1$ chains, and other $d_{J}<1$ chains had $T_{\text {orb }}$ values with relatively large $\chi^{2}$ values, while their $d_{J}<1$ counterparts generated no systems with stable orbits. As a result, these stable orbit, $d_{J}>1$, receive a higher than average weighting, but they still account for only $0.3 \%$ of the total weight. Thus, the $d_{J}>1$ models are excluded at $99.7 \%$ confidence.

\section{BAYESIAN ANALYSIS OF THE OGLE-2006-BLG-109L PLANETARY SYSTEM}

In Section 8, we described how we could convert a single set of light-curve model parameters into a one-parameter family of complete orbital solutions for both planets. We now apply this method to determine the orbital parameters of the OGLE-2006-BLG-109Lb,c planetary system. We do not apply the circular orbit constraint for the Saturn-mass planet to these calculations, but we do constrain the brightness of the lens star using the mass and distance derived from Equations (13) and (14), and dereddened $H$-band brightness of the lens star given by Equation (16) using an analytic model of the 
$H$-band mass-luminosity relation based on Kroupa \& Tout (1997), Delfosse et al. (2000), and Henry \& McCarthy (1993). Our basic method here is the MCMC, but there are several complications that must be addressed.

The first complication is that there are several relatively flat directions in parameter space that are not efficiently probed by the MCMC calculations, and efficiency is quite important because these calculations are rather CPU intensive. We perform a set of MCMC calculations with the fifth orbital parameter, $T_{\text {orb }}$ (the orbital period of the assumed circular orbit) fixed to a series of values: $2000,2500,2857,3333,4000,5000$, $6667,10,000$, and 20,000 days. These runs were done for both $d_{J}<1$ and $d_{J}>1$ models, but most of the $d_{J}>1$ produced no stable orbits. The only exception was the $d_{J}>1$, $T_{\text {orb }}=10^{4}$ days run, which yielded a few stable orbital solutions, although these amounted to only about $0.03 \%$ of the orbits that were consistent with the fit parameters of this MCMC run. In contrast, the run with $d_{J}<1$ and $T_{\text {orb }}=5000$ days, which is closest to our best-fit circular orbit model had a $50 \%$ stability rate for orbits that were consistent with the model parameters or the MCMC run. Also, the runs with $T_{\text {orb }}$ at the extremes of the range considered had few acceptable orbits. The run with $d_{J}<1$ and $T_{\text {orb }}=20,000$ days produced no bound Saturn-mass planets, and the $d_{J}<1$ with $T_{\text {orb }}=2000$ days produced only $0.6 \%$ bound orbits. The best-fit model at this $T_{\text {orb }}$ value is also disfavored by $\Delta \chi^{2}=7.5$ compared to the best-fit $T_{\text {orb }}=2000$ day model, and $\chi^{2}$ grows rapidly with smaller $T_{\text {orb }}$. So, our range of 2000 days $\leqslant T_{\text {orb }} \leqslant 20,000$ days seems to cover the range of viable orbital accelerations. These different MCMC runs with different fixed $T_{\text {orb }}$ values are combined with different weightings of $e^{-\Delta \chi^{2} / 2}$ based on the best-fit $\chi^{2}$ for each $T_{\text {orb }}$ value.

There are additional uncertainties that are not accounted for in these MCMC runs, so we have included them in our integration over the MCMC results. As mentioned in Section 8, the model parameters only specify five of the six parameters needed to completely describe the orbits, so we integrate over all the orbits consistent with the orbital stability constraint for each link in each Markov chain. Also, the uncertainty in the derived angular radius of the source star, Equation (12), is not included in the MCMC runs, so we select $\theta_{*}$ from a Gaussian random distribution based on Equation (12) when we sum the results of the MCMC runs.

The MCMC runs also do completely sample the microlensing parallax parameter space due to the partial degeneracy between the orbital motion and parallax parameters. In order to ensure that the uncertainty in $\pi_{E}$ is not underestimated, we have found the best-fit models with $\pi_{E}$ fixed on a one-dimensional grid with no lens brightness constraint. This calculation yields results that are well fit by $\pi_{E}=0.338 \pm 0.037$. We add this as an additional uncertainty in the same way as the uncertainty in $\theta_{*}$.

One consideration that has very important consequences for the orbital parameter results is the proper application of the Bayesian prior distribution for the orbital parameters. The model parameters have been designed for convenience and calculational efficiency during the modeling calculations, but they also implicitly provide the Bayesian prior for the MCMC calculation, which is basically a Bayesian likelihood calculation. In fact, the Bayesian prior provided by these parameters is quite an unreasonable one. This must be corrected to a more reasonable prior distribution by computing the Jacobian determinant of the coordinate transformation between the parameters used for the MCMC calculation and a set of parameters that describes a reasonable prior distribution. We select a prior that is uniform in orbital phase, eccentricity, time of periapsis, and the logarithm of the semimajor axis and has a random orientation of the orbit. The derivatives needed for the Jacobian determinant are calculated numerically, and the results have been shown not to depend on the numerical derivative calculations. However, to avoid numerical difficulties with this calculation, we have had to exclude orbits with orbital semimajor axes $>800 \mathrm{AU}$. Virtually all the models in the MCMC runs must have three-dimensional star-planet separations that are $<10 \mathrm{AU}$, and there are no viable models with three-dimensional separations much larger than $10 \mathrm{AU}$, because the light-curve requires planet velocities that would result in an unbound planet if the three-dimensional separation at the time of the event was $\gg 10$ AU. So, these excluded models with $a>800 \mathrm{AU}$ all have the planet detected extremely close to periapsis, which makes them extremely unlikely, and thus their exclusion has no effect on the results (except to remove large numerical errors).

\subsection{Physical Parameters of the OGLE-2006-BLG-109L System}

The results of our MCMC calculations and Bayesian planetary parameters analysis are summarized in Tables 1 and 2 . Tables 1 shows the nonlinear fit parameters of the best-fit circular orbit model and the mean and rms of the models contributing to the MCMC Bayesian analysis. The subscripts 1,2, and 3 refer to the Jupiter-mass planet, the Saturn-mass planet, and the host star, respectively. Table 2 shows the median, and the $1 \sigma$ and $2 \sigma$ limits on the physical parameters of the planets and their host star. $D_{L}$ is the distance to the lens system. $M_{A}, m_{b}$, and $m_{c}$ are the masses of the host star, the Jupiter, and the Saturn, respectively. The semimajor axes and periods are given by $a_{b}$, $a_{c}, P_{b}$, and $P_{c}$. For the Saturn-mass planet, $c$, we also constrain the orbital eccentricity, $\epsilon_{c}$, the inclination angle, $i_{c}$, and the axis of inclination, $\alpha_{c}$. The final two parameters, $K_{b}$, and $K_{c}$, are the velocity amplitudes that are normally measured by the Doppler radial velocity method.

This analysis shows that the Saturn-mass planet, OGLE2006-BLG-109Lc, is most consistent with being in a loweccentricity orbit, like Saturn in our own solar system, but the constraints are not very tight, with $1 \sigma$ and $2 \sigma$ upper limits on $\epsilon_{c}$ of 0.32 and 0.62 , respectively.

Note that the parameters in this table assume that the Jupiter-like planet is in a circular orbit. The uncertainty in $a_{J}$, the semimajor axis of the Jupiter, would undoubtably be larger without this assumption. However, the constraints on the eccentricity, $\epsilon_{c}$, of the Saturn-like planet would probably be tighter and would likely favor somewhat smaller eccentricities, if we had allowed non-circular orbits for the Jupiter. The reason for this is that an eccentric orbit for the Jupiter would make stability more difficult for the Saturn, and would likely exclude some of the higher $\epsilon_{c}$ orbits that are (barely) allowed in the current analysis.

We have also done a similar analysis of the properties of the Saturn-mass planet without the assumption that the Jupitermass planet is in a coplanar orbit, and the results are presented in Table 3. These results are similar to, but slightly weaker than, the results with the coplanar orbit assumption shown in Table 2. This indicates that most of the constraints on the Saturn-mass planet's orbit are likely to be due to the velocity components in the plane of the sky that have been measured with some precision from the microlensing light curve. 


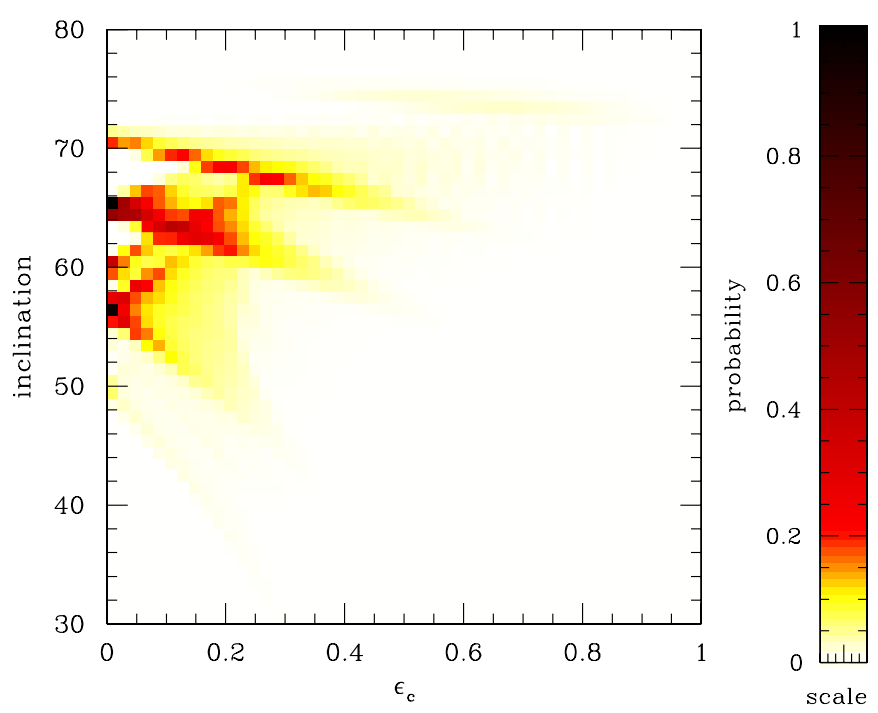

Figure 10. Probability distribution for the orbit of the Saturn-mass planet, OGLE-2006-BLG-Lc, is displayed in the eccentricity, $\epsilon_{c}$, vs. inclination angle, $i_{c}$, plane. The sparse sampling of the $T_{\text {orb }}$ parameter in the MCMC calculations is partially responsible for the distinct high probability regions in parameter space.

Table 3

Physical Parameters without Coplanar Assumption

\begin{tabular}{lcccccc}
\hline \hline Parameter & Units & \multicolumn{5}{c}{ Parameter Limits } \\
\cline { 3 - 7 } & & $-2 \sigma$ & $-1 \sigma$ & Median & $+1 \sigma$ & $+2 \sigma$ \\
\hline$a_{c}$ & AU & 2.5 & 3.3 & 4.8 & 8.4 & 15.6 \\
$P_{c}$ & Years & 5.5 & 8.0 & 14.8 & 32 & 81 \\
$\epsilon_{c}$ & & 0.01 & 0.07 & 0.27 & 0.65 & 0.85 \\
$i_{c}$ & deg & 37 & 51 & 62 & 72 & 75 \\
$K_{c}$ & $\mathrm{~m} \mathrm{~s}^{-1}$ & 2.6 & 3.6 & 4.2 & 5.0 & 5.3 \\
\hline
\end{tabular}

Some characteristics of the Saturn-mass planetary orbits that are consistent with the light curve are shown in Figure 10, which shows the probability distribution in the eccentricity, $\epsilon_{c}$, versus inclination, $i_{c}$, plane. Some of the structure in this plot is a result of the computational shortcut we have taken by running our MCMC runs with the model parameter $T_{\text {orb }}$ fixed at a fairly sparse set of values. However, the highest probability peaks in this distribution at $\epsilon_{c} \simeq 0$ and $i_{c} \simeq 56^{\circ}$ and $i_{c} \simeq 66^{\circ}$ have a different cause. These represent the two orbital configurations for which the most likely two-dimensional velocity of the Saturn-mass planet can match a circular orbit (Dong et al. 2009a).

\section{LIMITS ON ADDITIONAL PLANETS AND LENS STARS}

We have determined limits on other planets in the OGLE2006-BLG-109L system using the method of Rhie et al. (2000). In order to properly apply this method, we must first check that the fit $\chi^{2}$ cannot be significantly improved by adding an additional planet. At present, there is no existing modeling code that can handle a lens with four masses and orbital motion, so do we not attempt such fits. Instead, we have inspected the peak region of the light curve and determined that there is no localized region of the light curve where an additional, localized planetary signal could significantly improve the fit. Based on this, we have applied the Rhie et al. (2000) method with a threshold of $\Delta \chi^{2} \geqslant 60$. This allows us to exclude a Neptune-mass $\left(17 M_{\oplus}\right)$ planet in an annulus of projected separations ranging from
1.1 to $4.5 \mathrm{AU}$. If we assume coplanar orbits with the median $i_{c}=64^{\circ}$ inclination, we can de-project this to give an elliptical annulus that extends out to 2.5-10.3 AU. However, most of this exclusion region is probably already excluded by orbital stability constraints. This will generally be the situation for low mass planets in systems with gas-giant planets discovered near the Einstein radius.

The exclusion regions for additional giant planets are substantially larger. We can rule out another "Jupiter", with the same mass as OGLE-2006-BLG-109Lb over the projected separation range $0.5-10.5 \mathrm{AU}$. When we de-project this to get the exclusion region in the plane of the orbit, we find that the exclusion region extends from 1.1 AU to $24 \mathrm{AU}$ at the widest part of the elliptical annulus. The exclusion region rules out another "Jupiter" orbiting at a bit more than twice the orbital distance of the Saturn-mass planet. The exclusion region for another "Saturn," with the same mass as OGLE-2006-BLG-109Lc, extends about $72 \%$ as far as the exclusion region for another "Jupiter."

These limits on additional planets with a mass greater than $75 \%$ of Saturn's mass are essentially in the constant shear limit (Chang \& Refsdal 1979), where the light-curve deviation depends primarily on the shear parameter, $\gamma=q / d^{2}$, and for this event our limit corresponds to $\gamma<7 \times 10^{-5}$. This is the reason for our exclusion (in Section 6.2) of the possibility that the $H$-band flux attributed to the primary lens star could be from a companion to the lens, instead.

\section{DISCUSSION AND CONCLUSIONS}

We have presented the complete analysis of the OGLE-2006BLG-109Lb,c planetary system that was summarized by Gaudi et al. (2008), and we have explored the physics implications of the microlensing fit parameters. We have introduced a new method to determine the angular radius of the source star, $\theta_{*}$, which makes use of three color, VIH, light curve data to determine $\theta_{*}$ to a precision of $2.8 \%$. We then combine this measurement with the microlensing parallax parameter, $\pi_{E}$, determined from the microlensing light-curve model to yield a direct, geometric, measurement of the masses of the star and two planets in this planetary system. This is the only multiple planet system with measured masses for the stars and planets. (The pulsar planet system PSR B1257+12 (Konacki \& Wolszczan 2003) has measured planet masses, but the mass of the host neutron star is assumed.) The mass measurement of the host star is confirmed by Keck AO images, which detect the planetary host star and measure its $H$-band magnitude to be $H_{L}=17.17 \pm 0.05$.

These results, plus our more sophisticated modeling, including terrestrial parallax, confirm the results of Gaudi et al. (2008) that this system resembles a smaller version of our own solar system with a primary about half the mass of the Sun orbited by planets slightly smaller than Jupiter and Saturn in a similar arrangement with the Saturn-like planet outside. Their semimajor axes are about half of those of Jupiter and Saturn. This similarity to Jupiter and Saturn is even greater if we consider the configuration of the system during the process of planet formation at an age of $\sim 1$ million years, when nuclear fusion does not yet dominate the host star's energy production. At this time, the stellar luminosity is thought to scale as $\sim M^{2}$ (Burrows et al. 1993, 1997, G. Kennedy \& C. Lada, 2007, private communication). In the core accretion theory, the most massive planets are thought to form outside the "snow line" where it is cold enough for water-ice to form (Ida \& Lin 2004; Lecar et al. 2006; Kennedy et al. 2006; Kennedy \& Kenyon 2008), and this 
predicted $\sim M^{2}$ dependence of the proto-star luminosity implies that the location of the "snow line" should scale linearly with stellar mass. So, if our solar system's snow line was at 2.7 AU, which is slightly larger than half of Jupiter's semimajor axis, then we estimate that the snow line for OGLE-2006-BLG-109L should have been at $1.4 \mathrm{AU}$, which is slightly larger than half the semimajor axis of its Jupiter-analog planet.

Due to the relatively long, 11 day, duration of the signal of the Saturn-analog planet, the light-curve tightly constrains four of the six parameters that are needed to describe the planetary orbit. A fifth orbital parameter is weakly constrained. We use these light-curve constraints in an MCMC analysis, along with orbital stability constraints for the combined Jupiter/Saturn-analog system, to determine the constraints on the orbital parameters implied by the light-curve model. Assuming coplanar orbits, we find an orbital inclination of $i_{c}=64^{\circ}{ }_{-8^{\circ}}$, with a $2 \sigma$ lower limit of $49^{\circ}$. Because the lens star is relatively bright and brighter than the source, this means that it should be possible to confirm at least the inner, Jupiter-analog, planet with Doppler radial velocity measurements. While the host star at $H_{L}=17.17 \pm 0.05$ is much fainter than the host star for any known planet discovered with the radial velocity method, it seems reasonable to expect that the next generation of very large telescopes (Crampton et al. 2009; Hook 2009; Gilmozzi $\&$ Spyromilio 2009), combined with a high-throughput, highresolution spectrograph (Mayor et al. 2003) would be able to detect the radial velocity signal of the Jupiter-analog planet with a radial velocity amplitude of $K_{b}=17.4_{-1.1}^{+1.3} \mathrm{~m} \mathrm{~s}^{-1}$. This would be a challenging measurement for these extremely large telescopes, but our prior knowledge of the planetary system will minimize the number of observations that would be required. It is likely to be at least a decade and perhaps two decades before such measurements are possible, but this is much sooner than the $\gtrsim 10^{6} \mathrm{yr}$ that we would likely have to wait before either of these planets reveals themselves again via microlensing.

With our measurements of the planetary masses and constraints on the orbital parameters, OGLE-2006-BLG-109Lb,c is arguably the best constrained multiplanet system to orbit a main sequence star, other than the Sun. None of the other known multiple exoplanet systems has measured masses, and radial velocity measurements generally do not constrain more parameters of the planetary orbits than we have constrained for OGLE-2006-BLG-109c. On the other hand, radial velocity surveys often measure the eccentricity of the planets they detect more precisely than we have done, and the eccentricity is intrinsically more interesting than the parameters we have measured describing the orientation of the orbital plane of a single planet. Nevertheless, this is certainly the first planetary system found by microlensing in which the eccentricity of a planetary orbit is constrained.

It seems likely that we will be able to extract even more information on exoplanetary systems from microlensing events discovered in the near future. There have been two major telescope hardware improvements that have occurred since the discovery of OGLE-2006-BLG-109 that should significantly improve the light-curve coverage for future events. Later in the 2006 observing season, the MOA Collaboration brought its $1.8 \mathrm{~m}$ aperture MOA-II telescope (Hearnshaw et al. 2005) online equipped with the $2.2 \mathrm{deg}^{2}$ MOAcam-3 CCD camera (Sako et al. 2008). This allows an observing cadence as high as one observation every 15 minutes for the central Galactic bulge fields. OGLE has just completed an upgrade to its $1.4 \mathrm{deg}^{2}$ OGLE-IV camera (Udalski 2009) that will enable to increase its sampling rate in a similar fashion. If OGLE-IV had been in operation when OGLE-2006-BLG-109 was discovered, feature 1 in Figures 1 and 3 would have been recognized much sooner, which would likely have resulted in many more observations of this feature. Similarly, the larger MOA-II telescope and higher observing cadence enabled by MOA-cam-3 would have significantly improved the quantity and quality of the MOA data. As a result, it is very likely that if a similar event were detected today, we would be able to tightly constrain the fifth orbital motion parameter ( $T_{\text {orb }}$ in the parameterization we use here) and to weakly constrain the final orbital parameter. This would yield much tighter constraints on standard orbital parameters. So, for a fraction of the future exoplanets discovered by microlensing, we can expect better measurements of the orbital parameters than the ones we present here for OGLE-2006-BLG-109.

We thank Ian Thompson and Andy McWilliam for the reduction of the Magellan/MIKE spectrum of the lens star and its bright neighbor. D.P.B. was supported by grants AST0708890 from the NSF and NNX07AL71G from NASA. The OGLE project is partially supported by the Polish MNiSW grant N20303032/4275 to AU. S.N.'s, B.M.'s and K.H.C.'s work was performed under the auspices of the U.S. Department of Energy by Lawrence Livermore National Laboratory under contract DE-AC52-07NA27344. T.S. was supported by grant JSPS18749004. C.H. was supported by the National Research Fund of Korea 2009-0081561. B-GP and C-UL were supported by Korea Astronomy and Space Science Institute. Work by A.G. and S.D. was supported in part by grant AST-0757888 from the NSF. Work by B.S.G., A.G., and R.W.P. was supported in part by grant NNG04GL51G from NASA.

\section{REFERENCES}

Albrow, M. D., et al. 2009, MNRAS, 397, 2099

Alcock, C., et al. 1995, ApJ, 454, L125

Alcock, C., et al. 2001, ApJS, 136, 439

Alves, D. R., Rejkuba, M., Minniti, D., \& Cook, K. H. 2002, ApJ, 573, L51

Barnes, R., \& Greenberg, R. 2006, ApJ, 647, L163

Beaulieu, J.-P., et al. 2006, Nature, 439, 437

Bennett, D. P. 2008, in Exoplanets, ed. J. Mason (Berlin: Springer)

Bennett, D. P. 2009, ApJ, submitted (arXiv:0911.2703)

Bennett, D. P., Anderson, J., Bond, I. A., Udalski, A., \& Gould, A. 2006, ApJ, 647, L171

Bennett, D. P., \& Rhie, S. H. 1996, ApJ, 472, 660

Bennett, D. P., et al. 1993, BAAS, 25, 1402

Bennett, D. P., et al. 1999, Nature, 402, 57

Bennett, D. P., et al. 2008, ApJ, 684, 663

Bessell, M. S., \& Brett, J. M. 1988, PASP, 100, 1134

Bond, I. 2000, in ASP Conf. Proc. Ser. 216, Astronomical Data Analysis Software and Systems IX, ed. N. Manset, C. Veillet, \& D. Crabtree (San Francisco, CA: ASP), 555

Bond, I. A., et al. 2004, ApJ, 606, L155

Bozza, V. 1999, A\&A, 348, 311

Burrows, A., Hubbard, W. B., Saumon, D., \& Lunine, J. I. 1993, ApJ, 406, 158 Burrows, A., et al. 1997, ApJ, 491, 856

Butler, R. P., \& Marcy, G. W. 1996, ApJ, 464, L153

Cardelli, J. A., Clayton, G. C., \& Mathis, J. S. 1989, ApJ, 345, 245

Carpenter, J. M. 2001, AJ, 121, 2851

Cassan, A. 2008, A\&A, 491, 587

Chang, K., \& Refsdal, S. 1979, Nature, 282, 561

Claret, A. 2000, A\&A, 363, 1081

Claret, A., Diaz-Cordoves, J., \& Gimenez, A. 1995, A\&AS, 114, 247

Cohen, J. G., et al. 2009, ApJ, 699, 66

Crampton, D., Simard, L., \& Silva, D. 2009, in Astrophysics and Space Science

Proceedings, Science with the VLT in the ELT Era (The Netherlands: Springer), 279

Delfosse, X., Forveille, T., Ségransan, D., Beuzit, J.-L., Udry, S., Perrier, C., \& Mayor, M. 2000, A\&A, 364, 217

Diaz-Cordoves, J., Claret, A., \& Gimenez, A. 1995, A\&AS, 110, 329 
Dominik, M. 1999, A\&A, 349, 108

Dong, S., et al. 2006, ApJ, 642, 842

Dong, S., et al. 2007, ApJ, 664, 862

Dong, S., et al. 2009a, ApJ, 695, 970

Dong, S., et al. 2009b, ApJ, 698, 1826

Drimmel, R., \& Spergel, D. N. 2001, ApJ, 556, 181

Duquennoy, A., \& Mayor, M. 1991, A\&A, 248, 485

Gaudi, B. S., et al. 2008, Science, 319, 927

Gilmozzi, R., \& Spyromilio, J. 2009, in Astrophysics and Space Science Proceedings, Science with the VLT in the ELT Era (The Netherlands: Springer), 217

Gould, A. 1992, ApJ, 392, 442

Gould, A. 2000, ApJ, 542, 785

Gould, A. 2004, ApJ, 606, 319

Gould, A., Dong, S., Bennett, D. P., Bond, I. A., Udalski, A., \& Kozlowski, S. 2010, ApJ, 710, 1800

Gould, A., et al. 2006, ApJ, 644, L37

Gould, A., et al. 2009, ApJ, 698, L147

Griest, K., \& Safizadeh, N. 1998, ApJ, 500, 37

Han, C. 2005, ApJ, 629, 1102

Hardy, S. J., \& Walker, M. A. MNRAS, 276, L79

Hearnshaw, J. B., et al. 2005, arXiv:astro-ph/0509420

Henry, T. J., \& McCarthy, D. W., Jr. 1993, AJ, 106, 773

Hogan, E., Burleigh, M. R., \& Clarke, F. J. 2009, MNRAS, 396, 2074

Holtzman, J. A., et al. 1998, AJ, 115, 1946

Holz, D. E., \& Wald, R. M. 1996, ApJ, 471, 64

Hook, I. 2009, in Astrophysics and Space Science Proceedings, Science with the VLT in the ELT Era (The Netherlands: Springer), 225

Ida, S., \& Lin, D. N. C. 2004, ApJ, 616, 567

Janczak, J., et al. 2010, ApJ, 711, 731

Kennedy, G. M., \& Kenyon, S. J. 2008, ApJ, 673, 502

Kennedy, G. M., Kenyon, S. J., \& Bromley, B. C. 2006, ApJ, 650, L139

Kenyon, S. J., \& Hartmann, L. 1995, ApJS, 101, 117

Kervella, P., \& Fouqué, P. 2008, A\&A, 491, 855

Kervella, P., Thévenin, F., Di Folco, E., \& Ségransan, D. 2004, A\&A, 426, 297

Kilic, M., Gould, A., \& Koester, D. 2009, ApJ, 705, 1219

Konacki, M., \& Wolszczan, A. 2003, ApJ, 591, L147

Kroupa, P., \& Tout, C. A. 1997, MNRAS, 287, 402

Kubas, D., et al. 2008, A\&A, 483, 317

Lecar, M., Podolak, M., Sasselov, D., \& Chiang, E. 2006, ApJ, 640, 1115

Marcy, G. W., \& Butler, R. P. 1996, ApJ, 464, L147
Marshall, D. J., Robin, A. C., Reylé, C., Schultheis, M., \& Picaud, S. 2006, A\&A, 453,635

Mayor, M., \& Queloz, D. 1995, Nature, 378, 355

Mayor, M., et al. 2003, ESO Messenger, 114, 20

Metropolis, N., Rosenbluth, A. W., Rosenbluth, M. N., Teller, A. H., \& Teller, E. 1953, J. Chem. Phys., 21, 1087

Mullally, F., Winget, D. E., De Gennaro, S., Jeffery, E., Thompson, S. E., Chandler, D., \& Kepler, S. O. 2008, ApJ, 676, 573

Nishiyama, S., et al. 2006, ApJ, 638, 839

Poindexter, S., Afonso, C., Bennett, D. P., Glicenstein, J.-F., Gould, A., Szymański, M. K., \& Udalski, A. 2005, ApJ, 633, 914

Rattenbury, N. J., Bond, I. A., Skuljan, J., \& d Yock, P. C. M. 2002, MNRAS, 335,159

Refsdal, S. 1966, MNRAS, 134, 315

Reid, I. N., Gizis, J. E., \& Hawley, S. L. 2002, AJ, 124, 2721

Rhie, S. H. 2002, arXiv:astro-ph/0202294

Rhie, S. H., \& Bennett, C. S. 2009, MNRAS, submitted (arXiv:0911.3050)

Rhie, S. H., \& Bennett, D. P. 1996, Nucl. Phys. B Proc. Supp., 51, 86

Rhie, S. H., et al. 2000, ApJ, 533, 378

Sackmann, I.-J., Boothroyd, A. I., \& Kraemer, K. E. 1993, ApJ, 418, 457

Sako, T., et al. 2008, Exp. Astron., 22, 51

Salaris, M., \& Girardi, L. 2002, MNRAS, 337, 332

Schechter, P. L., Mateo, M., \& Saha, A. 1993, PASP, 105, 1342

Sigurdsson, S., Richer, H. B., Hansen, B. M., Stairs, I. H., \& Thorsett, S. E. 2003, Science, 301, 193

Skrutskie, M. F., et al. 2006, AJ, 131, 1163

Smith, M. C., Mao, S., \& Paczyński, B. 2003, MNRAS, 339, 925

Stetson, P. B. 1994, PASP, 106, 250

Sumi, T., et al. 2006, ApJ, 636, 240

Udalski, A. 2003, Acta Astron., 53, 291

Udalski, A. 2009, in ASP Conf. Ser. 403, The Variable Universe: A Celebration of Bohdan Paczyński (San Francisco, CA: ASP), 110

Udalski, A., Szymański, M., Kałużny, J., Kubiak, M., Mateo, M., Krzmiński, W., \& Paczyński, B. 1994, Acta Astron., 44, 227

Udalski, A., et al. 2002, Acta Astron., 52, 217

Udalski, A., et al. 2005, ApJ, 628, L109

Wambsganss, J. 1997, MNRAS, 284, 172

Wright, J. T., Marcy, G. W., Butler, R. P., Vogt, S. S., Henry, G. W., Isaacson, H., \& Howard, A. W. 2008, ApJ, 683, L63

Yoo, J., et al. 2004, ApJ, 603, 139

Zoccali, M., et al. 2000, ApJ, 530, 418 